CIRJE-F-918

\title{
Benchmarked Empirical Bayes Methods in Multiplicative Area-level Models with Risk Evaluation
}

\author{
Malay Ghosh \\ University of Florida \\ Tatsuya Kubokawa \\ The University of Tokyo \\ Yuki Kawakubo \\ Graduate School of Economics, The University of Tokyo \\ February 2014
}

CIRJE Discussion Papers can be downloaded without charge from:

http://www.cirje.e.u-tokyo.ac.jp/research/03research02dp.html

Discussion Papers are a series of manuscripts in their draft form. They are not intended for circulation or distribution except as indicated by the author. For that reason Discussion Papers may not be reproduced or distributed without the written consent of the author. 


\title{
Benchmarked Empirical Bayes Methods in Multiplicative Area-level Models with Risk Evaluation
}

\author{
Malay Ghosh*, Tatsuya Kubokawa ${ }^{\dagger}$ and Yuki Kawakubo ${ }^{\ddagger}$ \\ University of Florida and University of Tokyo
}

February 10, 2014

\begin{abstract}
The paper develops empirical Bayes and benchmarked empirical Bayes estimators of positive small area means under multiplicative models. A simple example will be estimation of per capita income for small areas. It is now well-understood that small area estimation needs explicit, or at least implicit use of models. One potential difficulty with model-based estimators is that the overall estimator for a larger geographical area based on (weighted) sum of the model-based estimators is not necessarily identical to the corresponding direct estimator, such as the overall sample mean. One way to fix such a problem is the so-called benchmarking approach which modifies the model-based estimators to match the aggregate direct estimator.

Benchmarked hierarchical and empirical Bayes estimators have proved to be particularly useful in this regard. However, while estimating positive small area parameters, the conventional squared error or weighted squared loss subject to the usual benchmark constraint does not necessarily produce positive estimators. Hence, it is necessary to seek other meaningful losses to alleviate this problem.

In this paper, we consider the transformed Fay-Herriot model as a multiplicative model for estimating positive small area means, and suggest a weighted KullbackLeibler divergence as a loss function. We have found out that the resulting Bayes estimator is the posterior mean and that the corresponding benchmarked Bayes and empirical Bayes estimators retain the positivity constraint.

The prediction errors of the suggested empirical Bayes estimators are investigated asymptotically, and their second-order unbiased estimators are provided. In addition, bootstrapped estimators of these prediction errors are also provided. The

*Department of Statistics, University of Florida, 102 Griffin-Floyd Hall, Gainesville, Florida 32611. E-Mail: ghoshm@stat.ufl.edu

${ }^{\dagger}$ Faculty of Economics, University of Tokyo, 7-3-1 Hongo, Bunkyo-ku, Tokyo 113-0033, JAPAN. E-Mail: tatsuya@e.u-tokyo.ac.jp

${ }^{\ddagger}$ Graduate School of Economics, University of Tokyo, 7-3-1 Hongo, Bunkyo-ku, Tokyo 113-0033, JAPAN. E-Mail: y.k.5.58.2010@gmail.com
\end{abstract}


performance of the suggested procedures is investigated through simulation as well as with an empirical study.

Key words and phrases: Asymptotic approximation, constrained Bayes, FayHerriot model, parametric bootstrap, second-order approximation, second-order unbiased.

\section{Introduction}

Bayesian and the related shrinkage methods have been extensively used and actively studied in small-area estimation. Since sample sizes of small areas are small, direct estimators, such as sample means have unacceptable estimation errors, and shrinkage estimators "borrowing strength" from other similar areas can often provide more reliable estimators with higher precision. However, one potential difficulty of the shrinkage estimators is that the overall estimator for a larger geographical area, which is often a weighted sum of the shrinkage estimators of individual small areas, is not necessarily equal to the corresponding overall direct estimator. One way to resolve this issue is the benchmarking approach, which modifies the shrinkage estimators so that one gets the same weighted aggregate direct estimator for larger geographical areas. To this end, benchmarked Bayes estimators have been addressed in a series of articles by You and Rao (2002, 2003), Datta, Ghosh, Steorts and Maples (2011), Steorts and Ghosh (2013), Ghosh and Steorts (2013) and Bell, Datta and Ghosh (2013). Non-Bayesian approach to this topic is available in Pfeffermann and Barnard (1991), Isaki, Tsay and Fuller (2000) and Wang, Fuller and Qu (2008) among others. For a good review on this topic, see Pfeffermann (2013).

In practice, one is often faced with estimation of positive area-level parameters, such as income, revenue, harvest, production, prices and others in small-areas. This is typical for the analysis of many official statistics and census data. A standard method for handling such cases is to make a logarithmic transformation of the data and use an additive linear mixed model such as the Fay-Herriot (1979) model to produce the small area estimates. This is equivalently described as a multiplicative model for the original positive data. Slud and Maiti (2006) discussed the difference between the additive and the multiplicative models through the second-order mean-squared error (MSE) criterion, i.e. correct MSE up to the $O\left(m^{-1}\right)$ term, where $m$ denotes the number of small areas.

For the problem of estimating positive means of small areas, we want to find hierarchical and empirical Bayes estimators subject to the constraint that the weighted sum of the estimators will equal the direct estimator for larger geographical areas. In the process of deriving the constrained Bayes estimators, we are faced with the following issues with a conventional approach:

(I) The standard loss function is the squared error loss $(\hat{\theta}-\theta)^{2}$, and estimators are evaluated in terms of the mean squared error (MSE). Although the squared error loss is useful for estimating real $\theta$, it is not necessarily an appropriate measure for estimation 
of positive $\theta$. In fact, the case of $\hat{\theta}<\theta$ is less penalized than that of $\hat{\theta}>\theta$ because of $\lim _{\hat{\theta} \rightarrow 0}(\hat{\theta}-\theta)^{2}=\theta^{2}$.

(II) The benchmark constraint considered here is the weighted sum of the original positive data. This setup is natural in practice. Under this restriction, the resulting constrained Bayes estimator relative to the squared error loss takes a negative value with a positive probability. For other conventional losses, the constrained Bayes estimators have complicated formulae or cannot be expressed in closed forms. Thus, the constrained Bayes estimators under the conventional losses are not so useful in practice.

As a feasible loss function which resolves these issues, we consider in this paper, a weighted Kullback-Leibler loss, a variant of the usual Kullback-Leibler loss, given by

$$
L_{m K L}(\theta, \hat{\theta})=\theta\{\hat{\theta} / \theta-\log (\hat{\theta} / \theta)-1\}=\hat{\theta}-\theta-\theta \log (\hat{\theta} / \theta)
$$

for estimating positive $\theta$. Clearly, $L_{m K L}(\theta, \hat{\theta})$ diverges when $\hat{\theta}$ goes to zero or infinity. It is interesting to note that the resulting Bayes estimator is the posterior mean, and as we will see later in Section 2, that the benchmarked Bayes estimator can be explicitly expressed, and hence can be readily implemented. Thus, the weighted Kullback-Leibler loss seems to be a feasible approach to resolve issues brought out in (I) and (II).

We have derived in this hierarchical empirical Bayes (HBEB) estimators which are different from the usual empirical Bayes (EB) estimators. While, for a large number of small areas, the difference between the two is relatively small, the former has an intrinsic appeal, since it is a Rao-Blackwellized version of the non-hierarchical Bayes estimator, and estimation of hyperparameters is left until the end. Specifically, for a normal prior, one can integrate out the mean parameter, and estimation of the prior variance occurs only at the final stage of estimation.

The paper is organized as follows: In Section 2, we describe the benchmark problem for the log-transformed Fay-Herriot model and demonstrate that the conventional loss functions cannot produce a simple and reasonable constrained Bayes estimator to satisfy the usual benchmark constraint. As an alternative, a weighted Kullback-Leibler loss is introduced, and the resulting constrained Bayes estimator is obtained in a closed form, while maintaining the necessary positivity constraint when transformed back. Also, in this section, we have derived the HBEB and the EB estimators, and have pointed out that the differ only in the $O\left(m^{-1}\right)$ terms, where $m$ is the number of small areas. In Section 3, we derive the second-order approximation of the risk function for the HBEB estimator. We also provide the second-order unbiased estimators of the risk function of the HBEB estimator via (i) an analytic method based on the Taylor expansion and (ii) a numerical method based on the parametric bootstrap. Section 4 contains some simulation results. Section 5 contains some approximations to the risks of the constrained HBEB estimators. Section 5 also contains the analysis of some real data. Some concluding remarks are made in Section 6. Proofs of some of the technical results are deferred to the appendix. 


\section{Constrained Bayes and empirical Bayes Estimation for Multiplicative Models}

\subsection{Transformed Fay-Herriot models and the resulting Bayes estimators}

In many surveys, the response consists of positive outcomes, such as income, revenue, harvest yield, production and many other quantities of interest. Their distributions are quite often positively skewed, and need suitable transformations for normality to hold. We will consider here, one such case, namely the multiplicative model (see e.g. Rao, 2003)

$$
y_{i}=\theta_{i} \eta_{i}, \quad i=1, \ldots, m,
$$

for positive response $y_{i}$. The objects of estimation are the $\theta_{i}$ for $i=1, \ldots, m$. It is convenient to use the log transformation

$$
z_{i}=\log y_{i}=\phi_{i}+\varepsilon_{i}, \quad i=1, \ldots, m,
$$

for $\phi_{i}=\log \theta_{i}$ and $\varepsilon_{i}=\log \eta_{i}$, where $\left(z_{i}, \phi_{i}\right), i=1, \ldots, m$ are mutually independent. The conditional distribution of $z_{i}$ given $\phi_{i}$ and the marginal distribution of $\phi_{i}$ are given by

$$
\begin{aligned}
z_{i} \mid \phi_{i}, \boldsymbol{\beta} & \sim \mathcal{N}\left(\phi_{i}, d_{i}\right), \\
\phi_{i} \mid \boldsymbol{\beta} & \sim \mathcal{N}\left(\boldsymbol{x}_{i}^{T} \boldsymbol{\beta}, \tau^{2}\right),
\end{aligned}
$$

where $\boldsymbol{x}_{i}$ 's are $p$-variate covariates, and $\boldsymbol{\beta}$ is a $p$-variate vector of regression coefficients. The parameter $\tau^{2}$ is assumed to be known for the moment, and will be estimated later. The $d_{i}$ 's are assumed to be known throughout to avoid non-identifiability. This is the wellknown Fay-Herriot (1979) model for $z_{i}$, but the parameters of interest are the $\theta_{i}=\exp \left(\phi_{i}\right)$, $i=1, \ldots, m$. We will write $\boldsymbol{z}=\left(z_{1}, \ldots, z_{m}\right)^{T}, \boldsymbol{\theta}=\left(\theta_{1}, \ldots, \theta_{m}\right)^{T}, \boldsymbol{\phi}=\left(\phi_{1}, \ldots, \phi_{m}\right)^{T}$, $\boldsymbol{X}^{T}=\left(\boldsymbol{x}_{1}, \ldots, \boldsymbol{x}_{m}\right)$, and assume $\operatorname{rank}(\boldsymbol{X})=p(<m)$ so that $\boldsymbol{X}^{T} \boldsymbol{X}$ is nonsingular. Also, we will write

$$
\begin{aligned}
\boldsymbol{\Sigma} & =\boldsymbol{\Sigma}\left(\tau^{2}\right)=\operatorname{diag}\left(d_{1}+\tau^{2}, \ldots, d_{m}+\tau^{2}\right), \\
\gamma_{i} & =\gamma_{i}\left(\tau^{2}\right)=d_{i} /\left(d_{i}+\tau^{2}\right), i=1, \ldots, m, \\
h_{i}(\omega) & =\exp \left(\boldsymbol{x}_{i}^{T} \boldsymbol{\beta}+\tau^{2} / 2\right), \quad \omega=\left(\boldsymbol{\beta}, \tau^{2}\right) .
\end{aligned}
$$

Concerning $\boldsymbol{\beta}$, we consider the following two cases:

(Case 1) Random coefficients for $\boldsymbol{\beta}$. It is assumed that $\boldsymbol{\beta}$ is a random vector of coefficients having the hierarchical prior distribution as $\boldsymbol{\beta} \sim$ uniform $\left(\mathbb{R}^{p}\right)$, the uniform distribution over $\mathbb{R}^{p}$.

(Case 2) $\boldsymbol{\beta}$. It is assumed that $\boldsymbol{\beta}$ is an unknown parameter which will be estimated from the marginal distribution of $\boldsymbol{z}$. 
Cases 1 and 2 lead to different posterior distributions of $\phi_{i}$. Let the generalized least squares (GLS) estimator of $\boldsymbol{\beta}$ for known $\tau^{2}$ be given by

$$
\widehat{\boldsymbol{\beta}}\left(\tau^{2}\right)=\left(\boldsymbol{X}^{T} \boldsymbol{\Sigma}^{-1} \boldsymbol{X}\right)^{-1} \boldsymbol{X}^{T} \boldsymbol{\Sigma}^{-1} \boldsymbol{z} .
$$

Then, we have the following proposition whose proof we omit.

Proposition 2.1 For Case 1, the posterior of $\phi_{i}$ given $\boldsymbol{z}$ is

$$
\phi_{i} \mid \boldsymbol{z}, \tau^{2} \sim \mathcal{N}\left(\widehat{\phi}_{i}^{H B}\left(\tau^{2}\right), k_{i}\left(\tau^{2}\right)\right)
$$

where

$$
\begin{aligned}
\widehat{\phi}_{i}^{H B}\left(\tau^{2}\right) & =\left(1-\gamma_{i}\right) z_{i}+\gamma_{i} \boldsymbol{x}_{i}^{T} \widehat{\boldsymbol{\beta}}\left(\tau^{2}\right), \\
k_{i}\left(\tau^{2}\right) & =\tau^{2} \gamma_{i}+\gamma_{i}^{2} \boldsymbol{x}_{i}^{T}\left(\boldsymbol{X}^{T} \boldsymbol{\Sigma}^{-1} \boldsymbol{X}\right)^{-1} \boldsymbol{x}_{i} .
\end{aligned}
$$

For Case 2, the posterior of $\phi_{i}$ given $\boldsymbol{z}$ is

$$
\phi_{i} \mid \boldsymbol{z}, \boldsymbol{\beta}, \tau^{2} \sim \mathcal{N}\left(\widehat{\phi}_{i}^{B}\left(\boldsymbol{\beta}, \tau^{2}\right), \tau^{2} \gamma_{i}\left(\tau^{2}\right)\right)
$$

where

$$
\widehat{\phi}_{i}^{B}\left(\boldsymbol{\beta}, \tau^{2}\right)=\left(1-\gamma_{i}\right) z_{i}+\gamma_{i} \boldsymbol{x}_{i}^{T} \boldsymbol{\beta} .
$$

The posterior means $E\left[\phi_{i} \mid \boldsymbol{z}\right]$ for Cases 1 and 2 are given respectively by $\widehat{\phi}_{i}^{H B}\left(\tau^{2}\right)$ and $\widehat{\phi}_{i}^{B}\left(\boldsymbol{\beta}, \tau^{2}\right)$. Substituting the GLS $\widehat{\boldsymbol{\beta}}\left(\tau^{2}\right)$ into $\widehat{\phi}_{i}^{B}\left(\boldsymbol{\beta}, \tau^{2}\right)$ yields the EB estimator

$$
\widehat{\phi}_{i}^{E B}\left(\tau^{2}\right)=\left(1-\gamma_{i}\right) z_{i}+\gamma_{i} \boldsymbol{x}_{i}^{T} \widehat{\boldsymbol{\beta}}\left(\tau^{2}\right),
$$

which is identical to the $\mathrm{HB}$ estimator $\widehat{\phi}_{i}^{H B}\left(\tau^{2}\right)$. This is not so for the $\theta_{i}$, since the posterior means of the $\theta_{i}$ for Cases $\mathbf{1}$ and $\mathbf{2}$ are given respectively by

$$
\begin{aligned}
\hat{\theta}_{i}^{H B}\left(\tau^{2}\right) & =E\left[\theta_{i} \mid \boldsymbol{z}, \tau^{2}\right]=E\left[\exp \left(\phi_{i}\right) \mid \boldsymbol{z}, \tau^{2}\right] \\
& =\exp \left\{\widehat{\phi}_{i}^{H B}\left(\tau^{2}\right)+k_{i}\left(\tau^{2}\right) / 2\right\}, \\
\hat{\theta}_{i}^{B}\left(\boldsymbol{\beta}, \tau^{2}\right) & =\hat{\theta}_{i}^{B}(\omega)=E\left[\theta_{i} \mid \boldsymbol{z}, \boldsymbol{\beta}, \tau^{2}\right] \\
& =\exp \left\{\widehat{\phi}_{i}^{B}\left(\boldsymbol{\beta}, \tau^{2}\right)+\tau^{2} \gamma_{i} / 2\right\},
\end{aligned}
$$

Substituting $\widehat{\boldsymbol{\beta}}\left(\tau^{2}\right)$ into $\hat{\theta}_{i}^{B}\left(\boldsymbol{\beta}, \tau^{2}\right)$ gives the EB estimator

$$
\hat{\theta}_{i}^{E B}\left(\tau^{2}\right)=\exp \left\{\widehat{\phi}_{i}^{E B}\left(\tau^{2}\right)+\tau^{2} \gamma_{i} / 2\right\}
$$

which is not identical to the HB estimator $\hat{\theta}_{i}^{H B}\left(\tau^{2}\right)$ given in (2.8). However, the difference between $\hat{\theta}_{i}^{H B}\left(\tau^{2}\right)$ and $\hat{\theta}_{i}^{E B}\left(\tau^{2}\right)$ is small as explained in the proposition given below. Assume the usual regularity conditions (see e.g. Prasad and Rao, 1990)

(C1) $\boldsymbol{X}^{T} \boldsymbol{X} / m$ converges to a positive definite matrix;

(C2) $\max _{1 \leq i \leq m} \boldsymbol{x}_{i}^{T}\left(\boldsymbol{X}^{T} \boldsymbol{X}\right)^{-1} \boldsymbol{x}_{i}=O\left(m^{-1}\right)$;

(C3) $0<d_{L} \leq d_{i} \leq d_{U}<\infty$ for all $i=1, \ldots, m$, where $d_{L}$ and $d_{U}$ do not depend on $m$. 
Proposition 2.2 Under the assumptions (A.1)-(A.3), it holds that $\hat{\theta}_{i}^{H B}\left(\tau^{2}\right)-\hat{\theta}_{i}^{E B}\left(\tau^{2}\right)=$ $O_{p}\left(m^{-1}\right)$ for large $m$.

In fact, the difference can be approximated as

$$
\begin{aligned}
\hat{\theta}_{i}^{H B}\left(\tau^{2}\right)-\hat{\theta}_{i}^{E B}\left(\tau^{2}\right) & =\hat{\theta}_{i}^{E B}\left(\tau^{2}\right)\left\{e^{\gamma_{i}^{2} \boldsymbol{x}_{i}^{T}\left(\boldsymbol{X}^{T} \boldsymbol{\Sigma}^{-1} \boldsymbol{X}\right)^{-1} \boldsymbol{x}_{i}}-1\right\} \\
& =\hat{\theta}_{i}^{E B}\left(\tau^{2}\right) \gamma_{i}^{2} \boldsymbol{x}_{i}^{T}\left(\boldsymbol{X}^{T} \boldsymbol{\Sigma}^{-1} \boldsymbol{X}\right)^{-1} \boldsymbol{x}_{i}+O_{p}\left(m^{-2}\right),
\end{aligned}
$$

which is of order $O_{p}\left(m^{-1}\right)$ due to the assumptions (C1), (C2) and (C3).

It is relevant to note that one may originally assume both $\boldsymbol{\beta}$ and $\tau^{2}$ to be known, and obtain the Bayes estimator $\hat{\theta}_{i}^{B}(\omega)$ of the $\theta_{i}$ and then obtain the EB estimator of $\theta_{i}$ by substituting the GLS estimator of $\boldsymbol{\beta}$ as before. We prefer the present approach due to the fact that

$$
\hat{\theta}_{i}^{H B}\left(\tau^{2}\right)=E\left[\theta_{i} \mid \boldsymbol{z}, \tau^{2}\right]=E\left[E\left[\theta_{i} \mid \boldsymbol{z}, \boldsymbol{\beta}, \tau^{2}\right] \mid \boldsymbol{z}, \tau^{2}\right]=E\left[\hat{\theta}_{i}^{B}(\omega) \mid \boldsymbol{z}, \tau^{2}\right]
$$

in the framework (2.2) with $\boldsymbol{\beta} \sim$ uniform $\left(\mathbb{R}^{p}\right)$. By the Rao-Blackwell theorem, $\hat{\theta}_{i}^{H B}\left(\tau^{2}\right)$ has smaller risk than that of $\hat{\theta}_{i}^{B}(\omega)$ for any convex loss.

\subsection{Benchmark problem and loss functions}

We now consider the benchmark problem of estimating the positive parameters $\theta_{i}$ 's by estimators $\hat{\theta}_{i}$ 's under the constraint

$$
\sum_{i=1}^{m} w_{i} \hat{\theta}_{i}=M(\boldsymbol{y}) \quad \text { and } \quad\left(\hat{\theta}_{1}, \ldots, \hat{\theta}_{m}\right)^{T} \in \Theta,
$$

where $w_{i}$ 's are weights such that $w_{i}>0$ and $\sum_{i=1}^{m} w_{i}=1$, while $\Theta=\left\{\left(\theta_{1}, \ldots, \theta_{m}\right)^{T} \mid \theta_{1}>\right.$ $\left.0, \ldots, \theta_{m}>0\right\}$. Here, $M(\boldsymbol{y})$ is a required constant or random variable like $M(\boldsymbol{y})=$ $\sum_{i=1}^{m} w_{i} y_{i}$. A reasonable method for deriving estimators satisfying the benchmark constraint (2.12) is the constrained Bayes procedure, which minimizes the posterior risk function subject to (2.12). A solution of the conditional optimality can be obtained with the method of Lagrange multipliers. Let $L(\boldsymbol{\theta}, \widehat{\boldsymbol{\theta}})$ be a loss function for estimating $\boldsymbol{\theta}=\left(\theta_{1}, \ldots, \theta_{m}\right)^{T}$ by an estimator $\widehat{\boldsymbol{\theta}}=\left(\hat{\theta}_{1}, \ldots, \hat{\theta}_{m}\right)^{T}$. Then, the Lagrange function is defined by

$$
L M(\widehat{\boldsymbol{\theta}}, \lambda)=E[L(\boldsymbol{\theta}, \widehat{\boldsymbol{\theta}}) \mid \boldsymbol{y}]+\lambda\left\{\sum_{i=1}^{m} w_{i} \hat{\theta}_{i}-M(\boldsymbol{y})\right\},
$$

where $\lambda$ is the Lagrange multiplier.

It is seen that the resulting constrained Bayes estimator depends on the choice of the loss function involved in the posterior risk. Our objective is to suggest an appropriate loss function so that the benchmarked estimators of the positive parameters $\theta_{i}$ are also positive. 
A standard loss function is the quadratic loss given by $L_{Q}(\boldsymbol{\theta}, \widehat{\boldsymbol{\theta}})=\sum_{i=1}^{m} \xi_{i}\left(\hat{\theta}_{i}-\theta_{i}\right)^{2}$ for positive constants $\xi_{i}$ 's. Then the Bayes estimator of $\theta_{i}$ is the posterior mean $E\left[\theta_{i} \mid y_{i}\right]$. The constrained Bayes estimator can be derived as the minimizer for the Lagrange function (2.13), and is given by

$$
\hat{\theta}_{i}^{C}=E\left[\theta_{i} \mid y_{i}\right]-\frac{w_{i} / \xi_{i}}{\sum_{j=1}^{m} w_{j}^{2} / \xi_{j}}\left(\sum_{j=1}^{m} w_{j} E\left[\theta_{j} \mid y_{j}\right]-M(\boldsymbol{y})\right) .
$$

A drawback of (2.14) is that $\hat{\theta}_{i}^{C}$ takes negative values with a positive probability. This suggests that we should derive a constrained Bayes estimator under the additional restriction $\hat{\theta}_{i}>0, i=1, \ldots, m$. However, such a constrained Bayes estimator cannot be expressed in a closed form. Another drawback of the quadratic loss $\left(\hat{\theta}_{i}-\theta_{i}\right)^{2}$ is that it penalizes $\hat{\theta}_{i}$ much less for $\hat{\theta}_{i}<\theta_{i}$ than for $\hat{\theta}_{i}>\theta_{i}$, since $\left(\hat{\theta}_{i}-\theta_{i}\right)^{2}$ converges to the finite value $\theta_{i}^{2}$ as $\hat{\theta}_{i} \rightarrow 0$. Although the quadratic loss function is an appropriate loss for estimation of a real valued parameter, it is not necessarily suitable for estimation of positive parameters.

An alternative loss is the log-transformed quadratic loss function given by $L_{T Q}(\boldsymbol{\theta}, \widehat{\boldsymbol{\theta}})=$ $\sum_{i=1}^{m} \xi_{i}\left(\log \hat{\theta}_{i}-\log \theta_{i}\right)^{2}$, which is quite natural since the multiplicative model (2.1) is transformed into the additive model via the log transformation. If the constraint is given by the geometric mean $\prod_{i=1}^{m} \hat{\theta}_{i}^{1 / m}=\prod_{i=1}^{m} y_{i}^{1 / m}$, then the benchmark problem in the multiplicative model can be reduced to the problem in the additive model with the constraint $m^{-1} \sum_{i=1}^{m} \log \hat{\theta}_{i}=m^{-1} \sum_{i=1}^{m} \log y_{i}$, and the constrained Bayes estimator and its properties can be studied along the lines of Datta et al. (2010). However, the constraint considered in this paper is the weighted mean constraint $\sum_{i=1}^{m} w_{i} \hat{\theta}_{i}=M(\boldsymbol{y})$, and the constrained Bayes estimator under this constraint cannot be derived explicitly. In fact, letting $\phi_{i}=\log \theta_{i}$ and $\widehat{\phi}_{i}=\log \hat{\theta}_{i}$, if we consider the problem of minimizing the posterior risk $\sum_{i=1}^{m} w_{i} E\left[\left(\widehat{\phi}_{i}-\phi_{i}\right)^{2} \mid \boldsymbol{z}\right]$ with respect to estimator $\widehat{\phi}_{i}$ subject to the constraint $\sum_{i=1}^{m} w_{i} \exp \left(\hat{\phi}_{i}\right)=M(\boldsymbol{y})$, the resulting constrained Bayes estimators $\hat{\phi}_{i}^{C}$ 's are solutions of the nonlinear equations

$$
M(\boldsymbol{y})\left\{\widehat{\phi}_{i}^{C}-E\left[\phi_{i} \mid z_{i}\right]\right\}=\exp \widehat{\phi}_{i}^{C} \sum_{j=1}^{m} w_{j}\left\{\widehat{\phi}_{j}^{C}-E\left[\phi_{j} \mid z_{j}\right]\right\}, \quad i=1, \ldots, m .
$$

Unfortunately, the solution cannot be expressed in a closed form, and we do not know about existence and uniqueness of the solution. Moreover, it is not easy to study any properties of these estimators. Thus, the loss function $L_{T Q}(\widehat{\boldsymbol{\theta}}, \boldsymbol{\theta})$ does not seem very suitable for the benchmark problem addressed here.

For estimating positive quantities, there are other options such as the Kullback-Leibler or the entropy loss and other related loss functions. James and Stein (1961) used such a loss in the context of estimation of a covariance matrix. The Kullback-Leibler (KL) loss is described as $L_{K L}(\boldsymbol{\theta}, \widehat{\boldsymbol{\theta}})=\sum_{i=1}^{m}\left\{\hat{\theta}_{i} / \theta_{i}-\log \left(\hat{\theta}_{i} / \theta_{i}\right)-1\right\}$, and the resulting Bayes estimator of $\theta_{i}$ is not the posterior mean, but the harmonic mean $1 / E\left[\theta_{i}^{-1} \mid z_{i}\right]$. The constrained Bayes estimator is then given by $\left(E\left[\theta_{i}^{-1} \mid y_{i}\right]+\lambda w_{i}\right)^{-1}$ where $\lambda$ is the solution of the equation $\sum_{j=1}^{m} w_{j} /\left(E\left[\theta_{j}^{-1} \mid y_{j}\right]+\lambda w_{i}\right)=M(\boldsymbol{y})$. 
We have investigated several conventional loss functions so far, but these losses cannot give us any convenient solution for finding benchmarked estimators of positive parameters. An alternative loss resulting in an easily interpretable estimator for the benchmark problem is given in the next subsection.

\subsection{Constrained HB estimator for a weighted KL loss}

Taking into account the drawbacks and/or inconveniences of typical loss functions as mentioned in the previous subsection, we suggest here an alternative loss function, a weighted KL loss, which is given by

$$
\begin{aligned}
L_{K L}^{w}(\boldsymbol{\theta}, \widehat{\boldsymbol{\theta}}) & =\sum_{i=1}^{m} w_{i} \theta_{i}\left\{\hat{\theta}_{i} / \theta_{i}-\log \left(\hat{\theta}_{i} / \theta_{i}\right)-1\right\} \\
& =\sum_{i=1}^{m} w_{i}\left\{\hat{\theta}_{i}-\theta_{i}-\theta_{i} \log \left(\hat{\theta}_{i} / \theta_{i}\right)\right\} .
\end{aligned}
$$

It is noted that $L_{K L}^{w}(\boldsymbol{\theta}, \widehat{\boldsymbol{\theta}}) \rightarrow \infty$ as $\hat{\theta}_{i} \rightarrow 0$ or as $\hat{\theta}_{i} \rightarrow \infty$. It is interesting to note that the resulting Bayes estimator of $\theta_{i}$ is the posterior mean $E\left[\theta_{i} \mid y_{i}\right]$. An appealing feature of the loss $L_{K L}^{w}(\boldsymbol{\theta}, \widehat{\boldsymbol{\theta}})$ is that the minimizer of the Lagrange function (2.13) can be easily obtained in an easily interpretable closed form. The resulting constrained Bayes estimator is a ratio adjusted estimator of the posterior mean, and is given by

$$
\hat{\theta}_{i}^{C}=E\left[\theta_{i} \mid y_{i}\right] \frac{M(\boldsymbol{y})}{\sum_{j=1}^{m} w_{j} E\left[\theta_{j} \mid y_{j}\right]} .
$$

This is a multiplicative form, and can be rewritten in a log-linear form as

$$
\log \hat{\theta}_{i}^{C}=\log E\left[\theta_{i} \mid y_{i}\right]-\left\{\log \sum_{j=1}^{m} w_{j} E\left[\theta_{j} \mid y_{j}\right]-\log M(\boldsymbol{y})\right\} .
$$

Thus, in this paper, we use the weighted KL loss $L_{K L}^{w}(\boldsymbol{\theta}, \widehat{\boldsymbol{\theta}})$. The properties of the weighted $\mathrm{KL}$ loss are summarized in the following proposition.

Proposition 2.3 Consider estimation of $\theta_{i}(>0)$ relative to the loss $L_{m K L}\left(\theta_{i}, \hat{\theta}_{i}\right)=\hat{\theta}_{i}-$ $\theta_{i}-\theta_{i} \log \left(\hat{\theta}_{i} / \theta_{i}\right)$. Then, the Bayes estimator of $\theta_{i}$ is given by the posterior mean $E\left[\theta_{i} \mid y_{i}\right]$ and the constrained Bayes estimator is provided as the natural ratio estimator (2.16). In the case when $E\left[\hat{\theta}_{i} / \theta_{i}\right]$ is a positive constant, the unbiased estimator of $\theta_{i}$ is the best among estimators $c \hat{\theta}_{i}$ for any constant $c$.

To see this, we write the risk of the estimator $c \hat{\theta}_{i}$ is as $E\left[c \hat{\theta}_{i}-\theta_{i}-\theta_{i} \log \left(c \hat{\theta}_{i} / \theta_{i}\right)\right]$, which is minimized by $c=1 / E\left[\hat{\theta}_{i} / \theta_{i}\right]$. Thus, when $E\left[\hat{\theta}_{i} / \theta_{i}\right]$ does not depend on $\theta_{i}$, the optimal estimator of $\theta_{i}$ within the given class of constant multiplier estimators of $\hat{\theta}_{i}$ is the unbiased estimator of $\theta_{i}$. 
When $\boldsymbol{\beta}$ is supposed to be distributed uniformly over $\mathbb{R}^{p}$, the posterior mean of $\theta_{i}$ is given by $\hat{\theta}_{i}^{H B}\left(\tau^{2}\right)=\exp \left\{\widehat{\phi}_{i}^{H B}\left(\tau^{2}\right)+k_{i}\left(\tau^{2}\right) / 2\right\}$ for $\widehat{\phi}_{i}^{H B}\left(\tau^{2}\right)=\left(1-\gamma_{i}\right) z_{i}+\gamma_{i} \boldsymbol{x}_{i}^{T} \widehat{\boldsymbol{\beta}}\left(\tau^{2}\right)$ as described in (2.9). Substituting the estimator into (2.16) yields the constrained HB (CHB) estimator

$$
\hat{\theta}_{i}^{C H B}\left(\tau^{2}\right)=\hat{\theta}_{i}^{H B}\left(\tau^{2}\right) \frac{M(\boldsymbol{y})}{\sum_{j=1}^{m} w_{j} \hat{\theta}_{j}^{H B}\left(\tau^{2}\right)} .
$$

Since $\tau^{2}$ is not known in practice, we need to estimate it. For the estimation of $\tau^{2}$, there are several methods proposed in the literature, which include the iterative method of moments estimator by Fay and Herriot (1979) and Morris (1983), the Prasad-Rao (1990) method of moment estimator, the maximum likelihood (ML) and the restricted maximum likelihood (REML) estimators proposed by Datta and Lahiri (2000) and the modified Prasad-Rao estimator proposed by Datta, Rao and Smith (2005). Some of them are given in the next section.

When $\tau^{2}$ is estimated by a consistent estimator $\hat{\tau}^{2}$, it can be substituted into $\hat{\theta}_{i}^{H B}\left(\tau^{2}\right)$ and $\hat{\theta}_{i}^{C H B}\left(\tau^{2}\right)$ to obtain the hierarchical empirical Bayes (HBEB) estimator $\hat{\theta}_{i}^{H B}\left(\hat{\tau}^{2}\right)$ and the corresponding constrained estimator $\hat{\theta}_{i}^{C H B}\left(\hat{\tau}^{2}\right)$. We will use the abbreviated notations $\hat{\theta}_{i}^{H B}$ and $\hat{\theta}_{i}^{C H B}$ instead of $\hat{\theta}_{i}^{H B}\left(\hat{\tau}^{2}\right)$ and $\hat{\theta}_{i}^{C H B}\left(\hat{\tau}^{2}\right)$ when there is no cofusion, and denote these estimators by $\mathrm{HB}$ and $\mathrm{CHB}$, respectively. We also use the notations $\widehat{\boldsymbol{\theta}}^{H B}=\left(\hat{\theta}_{1}^{H B}, \ldots, \hat{\theta}_{m}^{H B}\right)^{T}$ and $\widehat{\boldsymbol{\theta}}^{C H B}=\left(\hat{\theta}_{1}^{C H B}, \ldots, \hat{\theta}_{m}^{C H B}\right)^{T}$.

We can use the empirical Bayes estimator $\hat{\theta}_{i}^{E B}\left(\tau^{2}\right)$ given in (2.10) instead of $\hat{\theta}_{i}^{H B}\left(\tau^{2}\right)$. However, as shown in Proposition 2.2, the difference between the two estimators is quite small, and we omit the details for $\hat{\theta}_{i}^{E B}\left(\tau^{2}\right)$.

Remark 2.1 It may be interesting to note that the $\operatorname{loss} \theta(\hat{\theta} / \theta-\log (\hat{\theta} / \theta)-1)$ is connected to the $\operatorname{loss}(\log \hat{\theta}-\log \theta)^{2}$ through the loss function

$$
L_{m K L}(\theta, \hat{\theta}, t)=\frac{\theta^{t}}{t^{2}}\left\{(\hat{\theta} / \theta)^{t}-\log (\hat{\theta} / \theta)^{t}-1\right\}
$$

for real $t$. When $t \rightarrow 0, \lim _{t \rightarrow 0} L_{m K L}(\theta, \hat{\theta}, t)=(\log \theta-\log \theta)^{2} / 2$. The $\operatorname{loss} L_{m K L}(\theta, \hat{\theta})$ given in (1.1) corresponds to the case when $t=1$. For the general loss, the Bayes estimator of $\theta_{i}$ is given by

$$
\hat{\theta}_{t}^{B}=\left\{E\left[\theta^{t} \mid \boldsymbol{y}\right]\right\}^{1 / t}
$$

For $t=1$ and $t=-1$, the Bayes estimators correspond to the posterior mean $E[\theta \mid \boldsymbol{y}]$ and the posterior harmonic mean $1 / E\left[\theta^{-1} \mid \boldsymbol{y}\right]$, respectively. For $t \rightarrow 0$, the Bayes estimator tends to the geometric mean $\exp \{E[\log (\theta) \mid \boldsymbol{y}]\}$. 


\section{Evaluation and Estimation of Risk of the Empirical HB Estimator}

\subsection{A second-order approximation of the risk}

Our objective in this section is to find a second order correct (namely, correct up to $\left.O\left(m^{-1}\right)\right)$ expression for the risk of the empirical HB estimator $\hat{\theta}_{i}^{H B}\left(\hat{\tau}^{2}\right)$ under the loss $L_{m K L}\left(\theta_{i}, \hat{\theta}_{i}\right)$ given in (1.1), which is the $i$-th component of the loss given in (2.15). To this end, we need to add a couple of assumptions:

(C4) $\hat{\tau}^{2}(\boldsymbol{z}-\boldsymbol{X} \boldsymbol{\alpha})=\hat{\tau}^{2}(\boldsymbol{z})$ for any $\boldsymbol{\alpha}$, and $\hat{\tau}^{2}(-\boldsymbol{z})=\hat{\tau}^{2}(\boldsymbol{z})$.

(C5) The estimator $\hat{\tau}^{2}$ is consistent and $\hat{\tau}^{2}-\tau^{2}=O_{p}\left(m^{-1 / 2}\right)$. The bias and variance of $\hat{\tau}(\boldsymbol{z})$ are denoted by $\operatorname{Bias}\left(\hat{\tau}^{2}\right)$ and $\operatorname{Var}\left(\hat{\tau}^{2}\right)$, both of which are of order $O\left(\mathrm{~m}^{-1}\right)$.

Assumptions (C4) and (C5) are standard, and have been used for MSE calculations in Prasad and Rao (1990) (see also Rao, 2003). We now provide the following theorem in the log-transformed Fay-Herriot model (2.2). The proof is given in the Appendix.

Theorem 3.1 Under assumptions (C1)-(C5) and loss (1.1), the risk of the estimator $\hat{\theta}_{i}^{H B}\left(\hat{\tau}^{2}\right)$ of $\theta_{i}$ is

$$
\begin{aligned}
R_{\omega}\left(\hat{\theta}_{i}^{H B}\left(\hat{\tau}^{2}\right)\right)= & \frac{d_{i}}{2}\left(1-\gamma_{i}\right) h_{i}(\omega)+\frac{\gamma_{i}^{2}}{2} h_{i}(\omega) \boldsymbol{x}_{i}^{T}\left(\boldsymbol{X}^{T} \boldsymbol{\Sigma}^{-1} \boldsymbol{X}\right)^{-1} \boldsymbol{x}_{i} \\
& +\frac{\gamma_{i}^{2}}{8}\left\{\left(2-\gamma_{i}\right)^{2}+4 \gamma_{i} / d_{i}\right\} h_{i}(\omega) \operatorname{Var}\left(\hat{\tau}^{2}\right)+O\left(m^{-3 / 2}\right) .
\end{aligned}
$$

It may be noted that only the first term in the right hand side of $(3.1)$ is $O(1)$, while both the second and the third terms are of $O\left(\mathrm{~m}^{-1}\right)$.

The conditions (C4) and (C5) are satisfied by typical estimators of $\tau^{2}$. For example, the Prasad-Rao (1990) estimator for $\tau^{2}$ is given by $\hat{\tau}^{2 P R}=\max \left\{0, \hat{\tau}^{2 U}\right\}$, where

$$
\hat{\tau}^{2 U}=(m-p)^{-1}\left[\sum_{i=1}^{m}\left(z_{i}-\boldsymbol{x}_{i}^{T} \widetilde{\boldsymbol{\beta}}\right)^{2}-\sum_{i=1}^{m} d_{i}\left\{1-\boldsymbol{x}_{i}^{T}\left(\boldsymbol{X}^{T} \boldsymbol{X}\right)^{-1} \boldsymbol{x}_{i}\right\}\right],
$$

where $\widetilde{\boldsymbol{\beta}}=\left(\boldsymbol{X}^{T} \boldsymbol{X}\right)^{-1} \boldsymbol{X}^{T} \boldsymbol{z}$, the unweighted least square estimate of $\boldsymbol{\beta}$. This is useful due to its simplicity and analytical amenability. It is important to note that $P\left(\hat{\tau}^{2 P R}=0\right)$ converges to zero at an exponential rate when $m \rightarrow \infty$ (see Prasad and Rao, 1990). Also, $\operatorname{Bias}\left(\hat{\tau}^{2 U}\right)=0$ and $\operatorname{Var}\left(\hat{\tau}^{2 U}\right)=2 m^{-2} \operatorname{tr}\left[\boldsymbol{\Sigma}^{2}\right]+O\left(m^{-2}\right)$. Another well-known estimator $\hat{\tau}^{2 F H}$ is the estimator suggested by Fay and Herriot (1979) and it is given as the solution of the equation $L^{F H}\left(\hat{\tau}^{2 F H}\right)=0$, where

$$
L^{F H}\left(\tau^{2}\right)=\boldsymbol{z}^{T}\left\{\boldsymbol{\Sigma}\left(\tau^{2}\right)^{-1}-\boldsymbol{P}\left(\tau^{2}\right)\right\} \boldsymbol{z}-(m-p) .
$$

It follows from Datta, et al. (2005) that $\operatorname{Bias}\left(\hat{\tau}^{2 F H}\right)=2\left\{m \operatorname{tr}\left[\boldsymbol{\Sigma}^{-2}\right]-\left(\operatorname{tr}\left[\boldsymbol{\Sigma}^{-1}\right]\right)^{2}\right\} /\left(\operatorname{tr}\left[\boldsymbol{\Sigma}^{-1}\right]\right)^{3}+$ $O\left(m^{-3 / 2}\right)$ and $\operatorname{Var}\left(\hat{\tau}^{2 F H}\right)=2 m /\left(\operatorname{tr}\left[\boldsymbol{\Sigma}^{-1}\right]\right)^{2}+O\left(m^{-3 / 2}\right)$. For the ML and REML estimators, see Datta and Lahiri (2000). 
It is here pointed out that the empirical Bayes estimator $\hat{\theta}_{i}^{E B}\left(\hat{\tau}^{2}\right)$ given in $(2.10)$ has the same approximation as in (3.1). In fact, as seen from $(2.11)$, we have $\hat{\theta}_{i}^{H B}\left(\hat{\tau}^{2}\right)=$ $\hat{\theta}_{i}^{E B}\left(\hat{\tau}^{2}\right) \hat{\gamma}_{i}^{2} \boldsymbol{x}_{i}^{T}\left(\boldsymbol{X}^{T} \boldsymbol{\Sigma}\left(\hat{\tau}^{2}\right)^{-1} \boldsymbol{X}\right)^{-1} \boldsymbol{x}_{i}+O_{p}\left(m^{-2}\right)$. Then, the risk difference is written as

$$
\begin{aligned}
R_{\omega}\left(\hat{\theta}_{i}^{H B}\left(\hat{\tau}^{2}\right)\right)-R_{\omega}\left(\hat{\theta}_{i}^{E B}\left(\hat{\tau}^{2}\right)\right)= & E_{\omega}\left[\hat{\theta}_{i}^{H B}\left(\hat{\tau}^{2}\right)-\hat{\theta}_{i}^{E B}\left(\hat{\tau}^{2}\right)-\hat{\theta}_{i}^{B} \log \left(\hat{\theta}_{i}^{H B}\left(\hat{\tau}^{2}\right) / \hat{\theta}_{i}^{E B}\left(\hat{\tau}^{2}\right)\right)\right] \\
= & E_{\omega}\left[\hat{\theta}_{i}^{E B}\left(\hat{\tau}^{2}\right) \hat{\gamma}_{i}^{2} \boldsymbol{x}_{i}^{T}\left(\boldsymbol{X}^{T} \boldsymbol{\Sigma}\left(\hat{\tau}^{2}\right)^{-1} \boldsymbol{X}\right)^{-1} \boldsymbol{x}_{i}\right. \\
& \left.-\hat{\theta}_{i}^{B} \log \left(1+\hat{\gamma}_{i}^{2} \boldsymbol{x}_{i}^{T}\left(\boldsymbol{X}^{T} \boldsymbol{\Sigma}\left(\hat{\tau}^{2}\right)^{-1} \boldsymbol{X}\right)^{-1} \boldsymbol{x}_{i}\right)\right]+O\left(m^{-2}\right) \\
= & \left.E_{\omega}\left[\left\{\hat{\theta}_{i}^{E B}\left(\hat{\tau}^{2}\right)-\hat{\theta}_{i}^{B}\right\} \hat{\gamma}_{i}^{2} \boldsymbol{x}_{i}^{T}\left(\boldsymbol{X}^{T} \boldsymbol{\Sigma}\left(\hat{\tau}^{2}\right)^{-1} \boldsymbol{X}\right)^{-1} \boldsymbol{x}_{i}\right]+O\left(m^{-2}\right)\right),
\end{aligned}
$$

which is of $O\left(m^{-3 / 2}\right)$ since $\hat{\theta}_{i}^{E B}\left(\hat{\tau}^{2}\right)-\hat{\theta}_{i}^{B}=O_{p}\left(m^{-1 / 2}\right)$, where $\hat{\theta}_{i}^{B}=\hat{\theta}_{i}^{B}(\omega)$ is given in $(2.9)$.

Proposition 3.1 Under assumptions (C1)-(C5) and the loss (1.1), the risk of the empirical Bayes estimator $\hat{\theta}_{i}^{E B}\left(\hat{\tau}^{2}\right)$ has the same second-order approximation as in the risk of the empirical $H B$ estimator $\hat{\theta}_{i}^{H B}\left(\hat{\tau}^{2}\right)$.

\subsection{Second-order unbiased estimator of the risk}

There are two approaches to derivation of the second-order unbiased estimators for the risk, and we here provide the two methods.

We first estimate the risk based on the second-order approximation given in (3.1). Except for the first term in the right hand side of (3.1), all the other terms are of order $O\left(m^{-1}\right)$. Accordingly, one simply needs to estimate the unknown parameters $\boldsymbol{\beta}$ and $\tau^{2}$ involved in these terms by their estimators $\widehat{\boldsymbol{\beta}}\left(\hat{\tau}^{2}\right)$ and $\hat{\tau}^{2}$ given in the previous sections. Also, one estimates $\gamma_{i}=d_{i} /\left(d_{i}+\tau^{2}\right)$ by $\hat{\gamma}_{i}=d_{i} /\left(d_{i}+\hat{\tau}^{2}\right)$. The plug-in estimators for the terms with $O\left(\mathrm{~m}^{-1}\right)$ in (3.1) are also of order $O_{p}\left(\mathrm{~m}^{-1}\right)$ and the difference of these estimators and the terms they estimate are of the order $O_{p}\left(m^{-3 / 2}\right)$. Thus, the problem reduces to estimation of the first term $2^{-1} m_{i}(\omega)$ correct up to the order $O\left(m^{-1}\right)$ for $\omega=\left(\tau^{2}, \boldsymbol{\beta}\right)$, where

$$
m_{i}(\omega)=d_{i}\left(1-\gamma_{i}\right) \exp \left\{\boldsymbol{x}_{i}^{T} \boldsymbol{\beta}+\tau^{2} / 2\right\} .
$$

When $m_{i}(\omega)$ is estimated by the plug-in estimator $m_{i}(\hat{\omega})$ for $\hat{\omega}=\left(\widehat{\boldsymbol{\beta}}\left(\hat{\tau}^{2}\right), \hat{\tau}^{2}\right)$, the secondorder approximation of $E\left[m_{i}(\hat{\omega})\right]$ is given in the following lemma which will be proved in the Appendix.

Lemma 3.1 Under conditions (C1)-(C5), $E\left[m_{i}(\hat{\omega})\right]$ is approximated as

$$
\begin{aligned}
E\left[m_{i}(\hat{\omega})\right]= & m_{i}(\omega)+\frac{1}{2} \gamma_{i} h_{i}(\omega)\left\{\tau^{2} \boldsymbol{x}_{i}^{T}\left(\boldsymbol{X}^{T} \boldsymbol{\Sigma}^{-1} \boldsymbol{X}\right)^{-1} \boldsymbol{x}_{i}+\left(\tau^{2}+2 \gamma_{i}\right) \operatorname{Bias}\left(\hat{\tau}^{2}\right)\right. \\
& \left.+\left(-2 \gamma_{i}^{2} / d_{i}+\gamma_{i}+\tau^{2} / 4\right) \operatorname{Var}\left(\hat{\tau}^{2}\right)\right\}+O\left(m^{-3 / 2}\right) .
\end{aligned}
$$

Substituting the approximation (3.5) into (3.1) yields the second-order unbiased estimator of risk. 
Theorem 3.2 Under assumptions (C1)-(C5) and loss (1.1), the second-order unbiased estimator of risk of the empirical $H B$ estimator $\hat{\theta}_{i}^{H B}\left(\hat{\tau}^{2}\right)$ is given by

$$
\begin{aligned}
\widehat{R}\left(\hat{\theta}_{i}^{H B}\left(\hat{\tau}^{2}\right)\right)= & \frac{d_{i}}{2}\left(1-\hat{\gamma}_{i}\right) h_{i}(\hat{\omega})+\frac{1}{4} \hat{\gamma}_{i}\left(2 \hat{\gamma}_{i}-\hat{\tau}^{2}\right) h_{i}(\hat{\omega}) \boldsymbol{x}_{i}^{T}\left(\boldsymbol{X}^{T} \boldsymbol{\Sigma}^{-1}\left(\hat{\tau}^{2}\right) \boldsymbol{X}\right)^{-1} \boldsymbol{x}_{i} \\
& -\frac{1}{4} \hat{\gamma}_{i}\left(2 \hat{\gamma}_{i}+\hat{\tau}^{2}\right) h_{i}(\hat{\omega}) \widehat{\operatorname{Bias}}\left(\hat{\tau}^{2}\right) \\
& +\frac{\hat{\gamma}_{i}^{2}}{8}\left\{8 \hat{\gamma}_{i} / d_{i}+\left(2-\hat{\gamma}_{i}\right)^{2}-2-\hat{\tau}^{2} /\left(2 \hat{\gamma}_{i}\right)\right\} h_{i}(\hat{\omega}) \widehat{\operatorname{Var}}\left(\hat{\tau}^{2}\right),
\end{aligned}
$$

where $h_{i}(\hat{\omega})=\exp \left\{\boldsymbol{x}_{i}^{T} \widehat{\boldsymbol{\beta}}\left(\hat{\tau}^{2}\right)+\hat{\tau}^{2} / 2\right\}$. That is, $E\left[\widehat{R}\left(\hat{\theta}_{i}^{H B}\left(\hat{\tau}^{2}\right)\right)\right]=R\left(\omega, \hat{\theta}_{i}^{H B}\left(\hat{\tau}^{2}\right)\right)+O\left(m^{-3 / 2}\right)$.

We next provide another approach based on the method of parametric bootstrap. Consider the following multiplicative model: For $i=1, \ldots, m$, a positive observation $y_{i}^{*}$ follows the multiplicative model $y_{i}^{*}=\theta_{i}^{*} \eta_{i}^{*}$, where $\theta_{i}^{*}$ and $\eta_{i}^{*}$ are positive random variables which are described below. Let $z_{i}^{*}=\log y_{i}^{*}, \phi_{i}^{*}=\log \theta_{i}^{*}$ and $\varepsilon_{i}^{*}=\log \eta_{i}^{*}$. Then, $z_{i}^{*}$ has the following log linear model:

$$
\begin{aligned}
z_{i}^{*} & =\phi_{i}^{*}+\varepsilon_{i}^{*}, \quad \phi_{i}^{*}=\boldsymbol{x}_{i}^{T} \widehat{\boldsymbol{\beta}}\left(\hat{\tau}^{2}\right)+u_{i}^{*}, \\
u_{i}^{*} & \sim \mathcal{N}\left(0, \hat{\tau}^{2}\right), \quad \varepsilon_{i}^{*} \sim \mathcal{N}\left(0, d_{i}\right)
\end{aligned}
$$

where $\widehat{\boldsymbol{\beta}}\left(\hat{\tau}^{2}\right)$ and $\hat{\tau}^{2}=\hat{\tau}^{2}(\boldsymbol{y})$ are estimators based on $\boldsymbol{y}$ or $\boldsymbol{z}$ of the original model (2.1).

Let $J_{1}(\omega)=2^{-1} h_{i}(\omega) \tau^{2} \gamma_{i}$, and let $J_{2}(\omega)=R_{\omega}\left(\hat{\theta}_{i}^{H B}\left(\hat{\tau}^{2}\right)\right)-J_{1}(\omega)$. Then from Theorem 3.1 , it follows that $J_{1}(\omega)=O(1)$ and $J_{2}(\omega)=O\left(\mathrm{~m}^{-1}\right)$. We shall estimate $J_{1}$ and $J_{2}$ using the parametric bootstrap sample from (3.7). It is noted that the calculation of $\hat{\tau}^{2 *}=\hat{\tau}^{2 *}\left(\boldsymbol{y}^{*}\right)$ is the same as that of $\hat{\tau}^{2}=\hat{\tau}^{2}(\boldsymbol{y})$ except that $\hat{\tau}^{2 *}$ is calculated based on $\boldsymbol{y}^{*}$ instead of $\boldsymbol{y}$. Also, let $\widehat{\boldsymbol{\beta}}^{*}\left(\hat{\tau}^{2 *}\right)=\left(\boldsymbol{X}^{T} \boldsymbol{\Sigma}^{-1}\left(\hat{\tau}^{2 *}\right) \boldsymbol{X}\right)^{-1} \boldsymbol{X}^{T} \boldsymbol{\Sigma}^{-1}\left(\hat{\tau}^{2 *}\right) \boldsymbol{z}^{*}$. For $J_{1}$, we can use the arguments as in Butar and Lahiri (2003) to estimate it by

$$
J_{1}^{*}=2 J_{1}(\hat{\omega})-E_{*}\left[J_{1}\left(\hat{\omega}^{*}\right)\right]
$$

for $\hat{\omega}=\left(\widehat{\boldsymbol{\beta}}\left(\hat{\tau}^{2}\right), \hat{\tau}^{2}\right)$ and $\hat{\omega}^{*}=\left(\widehat{\boldsymbol{\beta}}^{*}\left(\hat{\tau}^{2 *}\right), \hat{\tau}^{2 *}\right)$, where $E_{*}[\cdot]$ denotes the expectation with respect to the model (3.7). For $J_{2}$, it can be estimated by

$$
\begin{aligned}
J_{2}^{*}= & -2^{-1} h_{i}(\hat{\omega}) \hat{\gamma}_{i}\left(2-\hat{\gamma}_{i}\right) \boldsymbol{x}_{i}^{T}\left(\boldsymbol{X}^{T} \boldsymbol{\Sigma}^{-1}\left(\hat{\tau}^{2}\right) \boldsymbol{X}\right)^{-1} \boldsymbol{x}_{i} \\
& +E_{*}\left[\hat{\theta}_{i}^{H B *}\left(\hat{\tau}^{2}\right)-\hat{\theta}_{i}^{B *}(\hat{\omega})-\hat{\theta}_{i}^{B *}(\hat{\omega}) \log \left(\hat{\theta}_{i}^{H B *}\left(\hat{\tau}^{2 *}\right) / \hat{\theta}_{i}^{H B *}\left(\hat{\tau}^{2}\right)\right)\right],
\end{aligned}
$$

where

$$
\begin{aligned}
\hat{\theta}_{i}^{H B *}\left(\hat{\tau}^{2}\right) & =\exp \left\{\left(1-\hat{\gamma}_{i}\right) z_{i}^{*}+\hat{\gamma}_{i} \boldsymbol{x}_{i}^{T} \widehat{\boldsymbol{\beta}}^{*}\left(\hat{\tau}^{2}\right)+k_{i}\left(\hat{\tau}^{2}\right) / 2\right\}, \\
\hat{\theta}_{i}^{H B *}\left(\hat{\tau}^{2 *}\right) & =\exp \left\{\left(1-\hat{\gamma}_{i}^{*}\right) z_{i}^{*}+\hat{\gamma}_{i}^{*} \boldsymbol{x}_{i}^{T} \widehat{\boldsymbol{\beta}}^{*}\left(\hat{\tau}^{2 *}\right)+k_{i}\left(\hat{\tau}^{2 *}\right)\right\}, \\
\hat{\theta}_{i}^{B *}(\hat{\omega}) & =\exp \left\{\left(1-\hat{\gamma}_{i}\right) z_{i}^{*}+\hat{\gamma}_{i} \boldsymbol{x}_{i}^{T} \widehat{\boldsymbol{\beta}}\left(\hat{\tau}^{2}\right)+\frac{d_{i}}{2}\left(1-\hat{\gamma}_{i}\right)\right\},
\end{aligned}
$$

for $\hat{\gamma}_{i}^{*}=d_{i} /\left(d_{i}+\hat{\tau}^{2 *}\right)$ and $\widehat{\boldsymbol{\beta}}^{*}\left(\hat{\tau}^{2}\right)=\left(\boldsymbol{X}^{T}\left(\boldsymbol{X}^{T} \boldsymbol{\Sigma}^{-1}\left(\hat{\tau}^{2}\right) \boldsymbol{X}\right)^{-1} \boldsymbol{X}^{T} \boldsymbol{\Sigma}^{-1}\left(\hat{\tau}^{2}\right) \boldsymbol{z}^{*}\right.$. Hence, we can get the following theorem. 
Theorem 3.3 Assume conditions (C1)-(C5). The second-order unbiased estimator of risk of the empirical $H B$ estimator $\hat{\theta}_{i}^{H B}\left(\hat{\tau}^{2}\right)$ relative to the loss (1.1) is given by

$$
\widehat{R}^{*}\left(\hat{\theta}_{i}^{H B}\left(\hat{\tau}^{2}\right)\right)=J_{1}^{*}+J_{2}^{*} .
$$

That is, $E\left[\widehat{R}^{*}\left(\hat{\theta}_{i}^{H B}\left(\hat{\tau}^{2}\right)\right)\right]=R\left(\omega, \hat{\theta}_{i}^{H B}\left(\hat{\tau}^{2}\right)\right)+O\left(m^{-3 / 2}\right)$.

\section{Simulation Study}

We begin by investigating the performance of second order unbiased estimators of the risk suggested in Section 3 by simulation. For the purpose, we adopt part of the simulation framework of Datta, et al. (2005) for our study. We consider the transformed Fay-Herriot model (2.1) with $m=15, \tau^{2}=1$ and two $d_{i}$-patterns: (a) $0.7,0.6,0.5,0.4,0.3$; (b) 2.0, 0.6, 0.5, 0.4, 0.2, which correspond to patterns (a) and (b) of Datta, et al. (2005). Pattern (a) is less variable in $d_{i}$-values, while pattern (b) has larger variability. There are five groups $G_{1}, \ldots, G_{5}$ and three small areas in each group. The sampling variances $d_{i}$ 's are the same for area within the same group. For the sake of computational simplicity, we handle the case $x_{i}=1$ and $\beta=1$, namely the model has only the constant term.

We prepare the true values of the risk of the empirical HB estimator $R\left(\omega, \hat{\theta}_{i}^{H B}\left(\hat{\tau}^{2}\right)\right)$ and the risk of $y_{i}, R\left(\omega, y_{i}\right)$ in advance, which can be computed based on 100,000 simulated data. The relative bias and the relative MSE of the estimator $\widehat{R}_{i}$ are given by

$$
\begin{aligned}
\operatorname{RBias}\left(\omega, \widehat{R}_{i}\right) & =100 \times E\left[\widehat{R}_{i}-R\left(\omega, \hat{\theta}_{i}^{H B}\left(\hat{\tau}^{2}\right)\right)\right] / R\left(\omega, \hat{\theta}_{i}^{H B}\left(\hat{\tau}^{2}\right)\right), \\
R M S E\left(\omega, \widehat{R}_{i}\right) & =100 \times E\left[\left\{\widehat{R}_{i}-R\left(\omega, \hat{\theta}_{i}^{H B}\left(\hat{\tau}^{2}\right)\right)\right\}^{2}\right] /\left\{R\left(\omega, \hat{\theta}_{i}^{H B}\left(\hat{\tau}^{2}\right)\right)\right\}^{2} .
\end{aligned}
$$

These values are computed as average values based on 10,000 simulation runs where the size of the bootstrap sample is 1,000. Further, those values are averaged over areas within groups $G_{i}, i=1, \ldots, 5$.

Let $\widehat{R}_{i}$ and $\widehat{R}_{i}^{*}$ be the estimators of the risk given in (3.6) and (3.11), respectively. Tables 1 and 2 report the true risks of $y_{i}$ and $\hat{\theta}_{i}^{H B}\left(\hat{\tau}^{2}\right)$, the second order approximation of $R\left(\omega, \hat{\theta}_{i}^{H B}\left(\hat{\tau}^{2}\right)\right)$ given in $(3.1)$, the estimates of $\widehat{R}_{i}, \widehat{R}_{i}^{*}$, the relative biases of $\widehat{R}_{i}, \widehat{R}_{i}^{*}$ and the relative MSE's of $\widehat{R}_{i}, \widehat{R}_{i}^{*}$, where the Prasad-Rao estimator is used in Table 1 , and the Fay-Herriot estimator is used in Table 2.

The tables tell us about some important features. Comparing the true risks of $y_{i}$ and $\hat{\theta}_{i}^{H B}\left(\hat{\tau}^{2}\right)$, one can see that $\hat{\theta}_{i}^{H B}\left(\hat{\tau}^{2}\right)$ improves on $y_{i}$, especially the improvement is significant at $G_{1}$ for pattern (b). Comparison of the true risks of $\hat{\theta}_{i}^{H B}\left(\hat{\tau}^{2}\right)$ in both tables tells us that $\hat{\theta}_{i}^{H B}\left(\hat{\tau}^{2}\right)$ with the Fay-Herriot estimator is slightly better than $\hat{\theta}_{i}^{H B}\left(\hat{\tau}^{2}\right)$ with the Prasad-Rao estimator, but the difference is not significant.

Comparing the risk estimates $\widehat{R}_{i}$ and $\widehat{R}_{i}^{*}$ via analytical and parametric bootstrap methods, one can see that both estimates are close each other except $G_{1}$ for pattern 
(b). The analytical estimator $\widehat{R}_{i}$ is better than $\widehat{R}_{i}^{*}$ in terms of dispersion based on MSE. Also, the biases of $\widehat{R}_{i}$ is smaller than $\widehat{R}_{i}^{*}$ except $G_{1}$. These observations suggest that the empirical HB estimator $\hat{\theta}_{i}^{H B}\left(\hat{\tau}^{2}\right)$ with the Fay-Herriot estimator is recommended, and that the analytical estimator $\widehat{R}_{i}$ is better than the parametric bootstrap method $\widehat{R}_{i}^{*}$, but the difference between the two procedures is not significant for pattern (a).

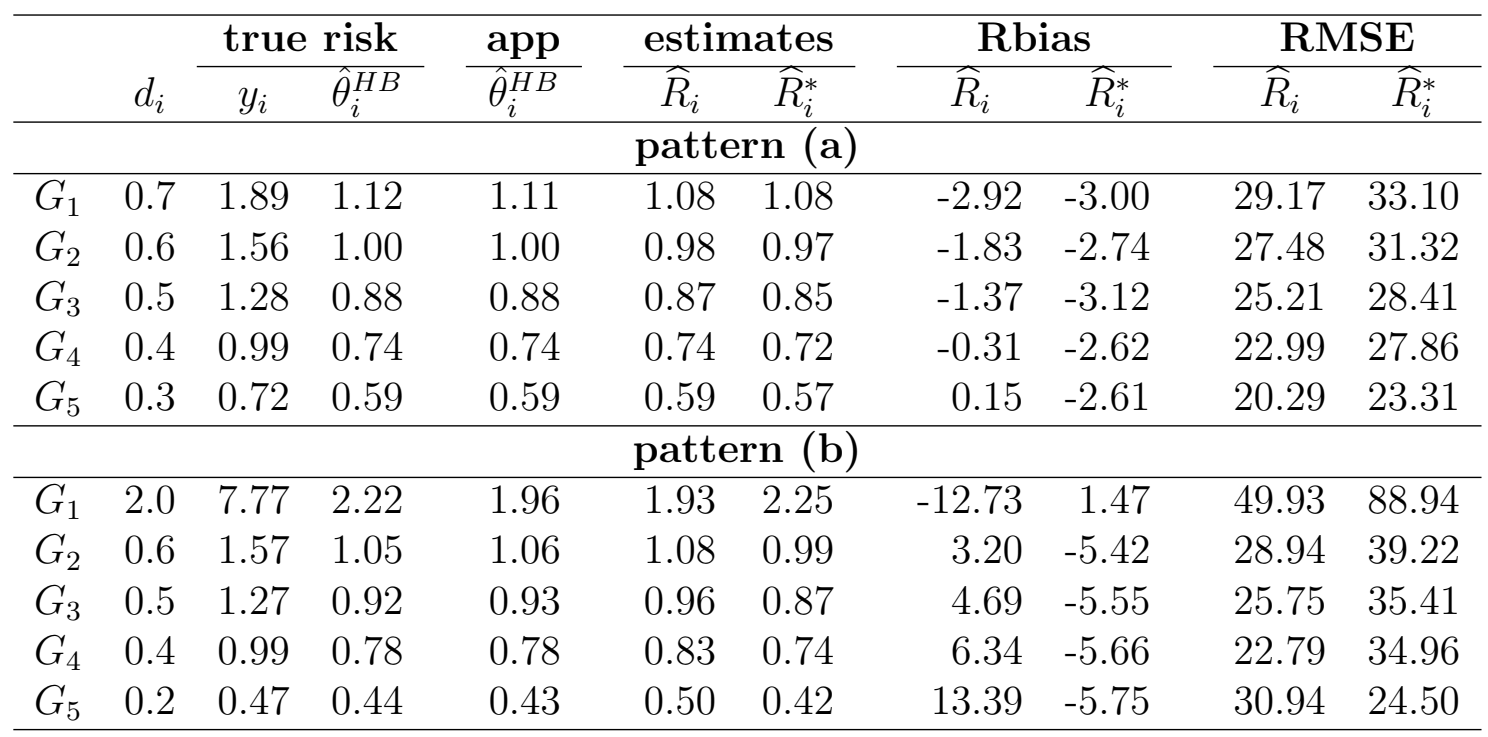

Table 1: Values of the true risks, the approximate risks, the risk estimates, the relative biases and the relative MSEs for the risk estimators for the Prasad-Rao estimator

\section{Approximation of Risk of the Empirical Constrained HB Estimator}

We now consider the approximation of the risk function of the empirical CHB estimator $\hat{\theta}_{i}^{C H B}$ given in (2.17), namely,

$$
\hat{\theta}_{i}^{C H B}\left(\hat{\tau}^{2}\right)=\hat{\theta}_{i}^{H B}\left(\hat{\tau}^{2}\right) \frac{M(\boldsymbol{y})}{\sum_{j=1}^{m} w_{j} \hat{\theta}_{j}^{H B}\left(\hat{\tau}^{2}\right)} .
$$

The risk relative to the $\operatorname{loss} L_{m K L}\left(\theta_{i}, \hat{\theta}_{i}\right)=\theta_{i}\left(\hat{\theta}_{i} / \theta_{i}-\log \left(\hat{\theta}_{i} / \theta_{i}\right)-1\right)$, which is the $i$-th component of the weighted KL loss, is written as

$$
\begin{aligned}
R\left(\omega, \hat{\theta}_{i}^{C H B}\left(\hat{\tau}^{2}\right)\right)= & R\left(\omega, \hat{\theta}_{i}^{H B}\left(\hat{\tau}^{2}\right)\right) \\
& -E\left[\hat{\theta}_{i}^{B}(\omega) \log \frac{M(\boldsymbol{y})}{\sum_{j=1}^{m} w_{j} \hat{\theta}_{j}^{H B}\left(\hat{\tau}^{2}\right)}\right]+E\left[\hat{\theta}_{i}^{H B}\left(\hat{\tau}^{2}\right)\left\{\frac{M(\boldsymbol{y})}{\sum_{j=1}^{m} w_{j} \hat{\theta}_{j}^{H B}\left(\hat{\tau}^{2}\right)}-1\right\}\right] \\
= & R\left(\omega, \hat{\theta}_{i}^{H B}\right)-K_{1}+K_{2} \text {. (say) }
\end{aligned}
$$




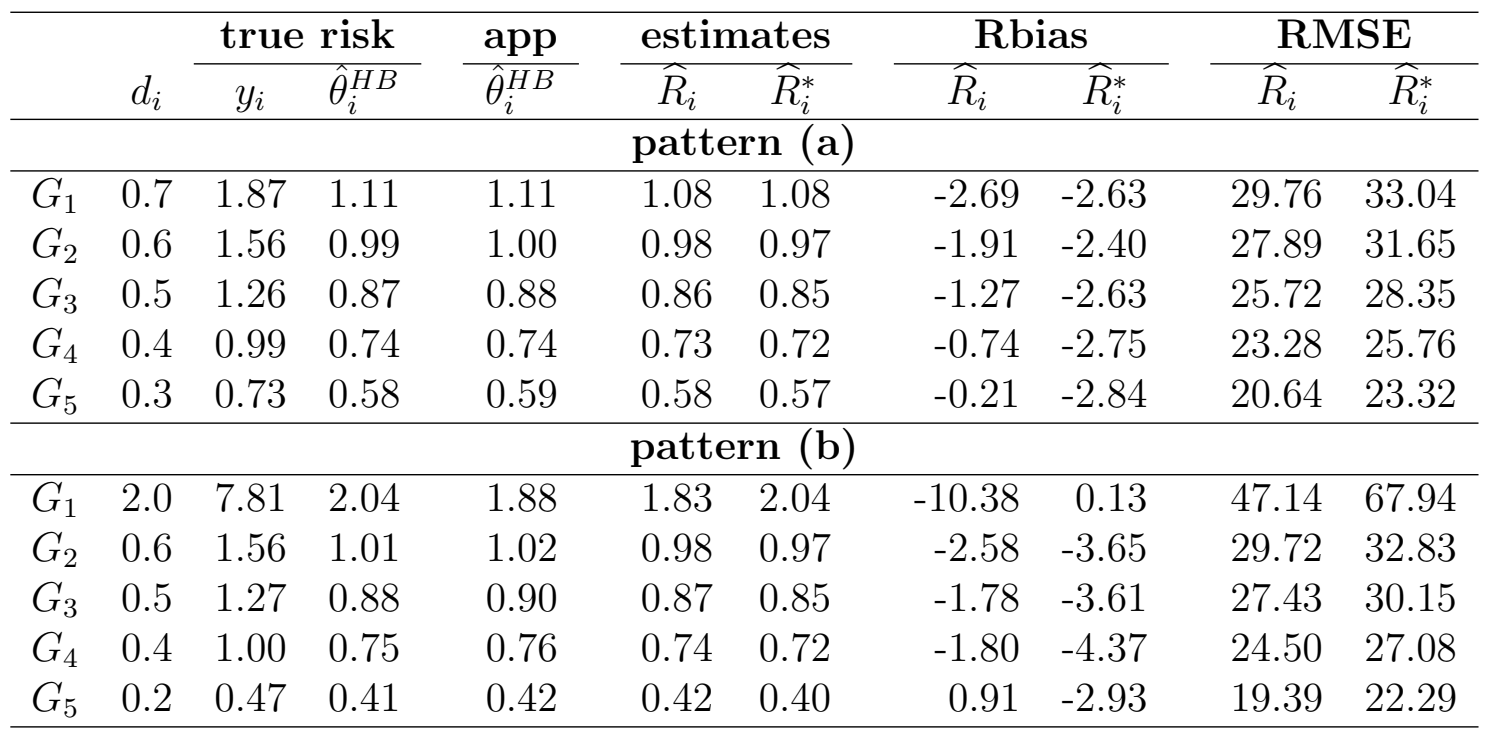

Table 2: Values of the true risks, the approximated risk, the risk estimates, the relative biases and the relative MSEs for the risk estimators for the Fay-Herriot estimator

Hereafter, we treat the natural target for benchmarking given by

$$
M(\boldsymbol{y})=\sum_{i=1}^{m} w_{i} y_{i}=\sum_{i=1}^{m} w_{i} e^{z_{i}}
$$

Then, $\sum_{i=1}^{m} w_{i} y_{i}$ and $\sum_{j=1}^{m} w_{j} \hat{\theta}_{j}^{H B}\left(\hat{\tau}^{2}\right)$ converge to different values as shown in Lemma 5.1 , which will be proved in the Appendix. Assume the following condition on $w_{i}$ :

(C6) The weight $w_{i}^{\prime}$ 's are nonnegative constants satisfying $\sum_{i=1}^{m} w_{i}=1$ and $\sum_{i=1}^{m} w_{i}^{2}=$ $O\left(m^{-1}\right)$.

Lemma 5.1 Under conditions (C1)-(C6), $\sum_{i=1}^{m} w_{i} y_{i}$ and $\sum_{i=1}^{m} w_{i} \hat{\theta}_{i}^{H B}\left(\hat{\tau}^{2}\right)$ are approximated as, for $h_{i}(\omega)$ given in (2.3),

$$
\begin{aligned}
\sum_{i=1}^{m} w_{i} y_{i} & =\sum_{i=1}^{m} w_{i} h_{i}(\omega) \exp \left\{d_{i} / 2\right\}+O_{p}\left(m^{-1 / 2}\right), \\
\sum_{i=1}^{m} w_{i} \hat{\theta}_{i}^{H B}\left(\hat{\tau}^{2}\right) & =\sum_{i=1}^{m} w_{i} h_{i}(\omega)+O_{p}\left(m^{-1 / 2}\right) .
\end{aligned}
$$

Lemma 5.1 means that

$$
\frac{\sum_{i=1}^{m} w_{i} y_{i}}{\sum_{i=1}^{m} w_{i} \hat{\theta}_{i}^{H B}\left(\hat{\tau}^{2}\right)}=\frac{\sum_{i=1}^{m} w_{i} h_{i}(\omega) \exp \left\{d_{i} / 2\right\}}{\sum_{i=1}^{m} w_{i} h_{i}(\omega)}+O_{p}\left(m^{-1 / 2}\right) .
$$

Noting that $E\left[\hat{\theta}_{i}^{B}(\omega)\right]=h_{i}(\omega)$ and $\lim _{m \rightarrow \infty} E\left[\hat{\theta}_{i}^{H B}\left(\hat{\tau}^{2}\right)\right]=h_{i}(\omega)$, we can see that

$$
\lim _{m \rightarrow \infty}\left\{-K_{1}+K_{2}\right\}=h_{i}(\omega)\left\{C_{i}(\omega)-\log C_{i}(\omega)-1\right\}
$$


where $C_{i}(\omega)=\sum_{i=1}^{m} w_{i} h_{i}(\omega) \exp \left\{d_{i} / 2\right\} / \sum_{i=1}^{m} w_{i} h_{i}(\omega)$.

Proposition 5.1 Assume conditions (C1)-(C6). Then,

$$
\lim _{m \rightarrow \infty}\left\{R\left(\omega, \hat{\theta}_{i}^{C H B}\left(\hat{\tau}^{2}\right)\right)-R\left(\omega, \hat{\theta}_{i}^{H B}\left(\hat{\tau}^{2}\right)\right)\right\}=h_{i}(\omega)\left\{C_{i}(\omega)-\log C_{i}(\omega)-1\right\},
$$

which is positive and of order $O(1)$.

Proposition 5.1 shows that the difference between the risk functions of $\hat{\theta}_{i}^{C H B}$ and $\hat{\theta}_{i}^{H B}$ is in the leading term, which implies that this difference is not negligible. Thus, we need to estimate how much the risk of $\hat{\theta}_{i}^{C H B}$ is inflated. We next provide the second-order unbiased estimator of the risk of $\hat{\theta}_{i}^{C H B}$ using the parametric bootstrap method, because the corresponding analytical second-order unbiased estimator based on the Taylor series expansion is harder to derive in this case.

The problem is how we should estimate $-K_{1}+K_{2}$. To this end, we shall rewrite it as $-K_{1}+K_{2}=E[\widehat{K}]+K_{3}$, where

$$
\begin{aligned}
\widehat{K} & =\hat{\theta}_{i}^{H B}\left(\hat{\tau}^{2}\right)\left\{\frac{\sum_{i=1}^{m} w_{i} y_{i}}{\sum_{j=1}^{m} w_{j} \hat{\theta}_{j}^{H B}\left(\hat{\tau}^{2}\right)}-\log \left(\frac{\sum_{i=1}^{m} w_{i} y_{i}}{\sum_{j=1}^{m} w_{j} \hat{\theta}_{j}^{H B}\left(\hat{\tau}^{2}\right)}\right)-1\right\}, \\
K_{3} & =E\left[\left\{\hat{\theta}_{i}^{H B}\left(\hat{\tau}^{2}\right)-\hat{\theta}_{i}^{B}(\omega)\right\} \log \left(\frac{\sum_{i=1}^{m} w_{i} y_{i}}{\sum_{j=1}^{m} w_{j} \hat{\theta}_{j}^{H B}\left(\hat{\tau}^{2}\right)}\right)\right] .
\end{aligned}
$$

Since $\widehat{K}$ is an exact unbiased estimator of $E[\widehat{K}]$, it is sufficient to provide a second-order unbiased estimator of $K_{3}$. We here estimate $K_{3}$ using the parametric bootstrap method. Let $y_{i}^{*}$ or $z_{i}^{*}, i=1, \ldots, m$, be random variables generated from the model (3.7). Based on the bootstrap sample $y_{i}^{*}$ s, we define the estimators

$$
K_{3}^{*}=E_{*}\left[\left\{\hat{\theta}_{j}^{H B *}\left(\hat{\tau}^{2 *}\right)-\hat{\theta}_{i}^{B *}(\hat{\omega})\right\} \log \frac{\sum_{i=1}^{m} w_{i} y_{i}^{*}}{\sum_{j=1}^{m} w_{j} \hat{\theta}_{j}^{H B *}\left(\hat{\tau}^{2 *}\right)} \mid \boldsymbol{y}\right],
$$

where $\hat{\theta}_{i}^{B *}(\hat{\omega})$ is defined at (3.10). We can see that $E\left[K_{3}^{*}\right]=K_{3}+O\left(m^{-3 / 2}\right)$ if $K_{3}$ is of order $O\left(\mathrm{~m}^{-1}\right)$. In the Appendix, we shall verify that $K_{3}=O\left(\mathrm{~m}^{-1}\right)$, and prove the following theorem.

Theorem 5.1 Assume conditions (C1)-(C6). Also, assume that for $i=1, \ldots, m$, and any $\tau^{2}, \hat{\tau}^{2}\left(\boldsymbol{z}+\tau^{2} \boldsymbol{e}_{i}\right)=\hat{\tau}^{2}(\boldsymbol{z})+O_{p}\left(m^{-1}\right)$, where $\boldsymbol{e}_{i}$ be an $m \times 1$ vector such that the $i$-th element is one and the others are zero. Let $M(\boldsymbol{y})=\sum_{i=1}^{m} w_{i} y_{i}$. Then, the second-order unbiased estimator of risk of the empirical CHB estimator $\hat{\theta}_{i}^{C H B}\left(\hat{\tau}^{2}\right)$ is given by

$$
\widehat{R}^{*}\left(\hat{\theta}_{i}^{C H B}\left(\hat{\tau}^{2}\right)\right)=\widehat{R}^{*}\left(\hat{\theta}_{i}^{H B}\left(\hat{\tau}^{2}\right)\right)+\widehat{K}+K_{3}^{*},
$$

where $\widehat{R}^{*}\left(\hat{\theta}_{i}^{H B}\left(\hat{\tau}^{2}\right)\right), \widehat{K}$ and $K_{3}^{*}$ are given in (3.11), (5.3) and (5.5), respectively. That is, $E\left[\widehat{R}^{*}\left(\hat{\theta}_{i}^{C H B}\left(\hat{\tau}^{2}\right)\right)\right]=R\left(\omega, \hat{\theta}_{i}^{C H B}\left(\hat{\tau}^{2}\right)\right)+O\left(m^{-3 / 2}\right)$. 
We now apply the empirical HB and CHB estimators, given in (2.8) and (5.1), to the data in the Survey of Family Income and Expenditure (SFIE) in Japan. Also we investigate the performances of the second-order unbiased estimators of the risks of the empirical HB and CHB estimators through analysis of the data.

In this study, we use the data of the disbursement item 'Education' in the survey in November, 2011. The average disbursement (scaled by 1,000 Yen) at each capital city of 47 prefectures in Japan is denoted by $y_{i}$ for $i=1, \ldots, 47$ with $m=47$, and each variance $d_{i}$ is calculated based on data of the disbursement 'Education' at the same city every November in the past ten years. Although the average disbursements in SFIE are reported every month, the sample sizes are around 100 for most prefectures, and data of the item 'Education' have high variability. On the other hand, we have data in the National Survey of Family Income and Expenditure (NSFIE) for 47 prefectures. Since NSFIE is based on much larger sample than SFIE, the average disbursements in NSEDI are more reliable, but this survey has been implemented every five years. In this study, we use the log-transformed data of the item 'Education' of NSFIE in 2009, which is denoted by $X_{i}$ for $i=1, \ldots, 47$.

For $i=1, \ldots, 47$, the observation $y_{i}$ follows the multiplicative model

$$
y_{i}=\theta_{i} \eta_{i}
$$

where $z_{i}=\log y_{i}, \phi_{i}=\log \theta_{i}$ and $\varepsilon_{i}=\log \eta_{i}$ follow the model given in (2.1). For $\tau^{2}$, we used the Fay-Herriot estimator, which yields $\hat{\tau}^{2 F H}=0.0520214$ in this example. The value of the ratio $\sum_{i=1}^{m} w_{i} y_{i} / \sum_{j=1}^{m} w_{j} \hat{\theta}_{j}^{H B}\left(\hat{\tau}^{2 F H}\right)$ is 1.0876 , and the empirical CHB estimates are obtained by multiplying the empirical HB estimates by 1.0876 . The sample size in each area (prefecture) is denoted by $n_{i}$. The estimators $\hat{\theta}_{i}^{H B}\left(\hat{\tau}^{2 F H}\right)$ and $\hat{\theta}_{i}^{C H B}\left(\hat{\tau}^{2 F H}\right)$ given in (2.8) and (5.1) with $\tau^{2}=\hat{\tau}^{2 F H}$ are denoted by HB and CHB, respectively, where $M(\boldsymbol{y})=\sum_{i=1}^{m} w_{i} y_{i}$ for $w_{i}=n_{i} / \sum_{j=1}^{47} n_{j}$. For the second-order unbiased estimators of the risk of $\hat{\theta}_{i}^{H B}$, the analytical estimator (3.6) and the parametric bootstrap alternative (3.11) are denoted by $R_{H B}$ and $R_{H B}^{*}$, respectively. The second-order unbiased estimator of the risk of $\hat{\theta}_{i}^{C H B}$ based on the parametric bootstrap is denoted by $R_{C H B}^{*}$.

Among 47 prefectures in Japan, we select the seven prefectures in the Kanto region around Tokyo, and Table 3 gives their values of $n_{i}, d_{i}, y_{i}, \mathrm{HB}, \mathrm{CHB}, R_{H B}, R_{H B}^{*}$ and $R_{C H B}^{*}$, where the values of $d_{i}, R_{H B}, R_{H B}^{*}$ and $R_{C H B}^{*}$ multiplied by 100 are reported. As seen by comparing the values given in Chiba and Tokyo, the empirical HB estimate shrinks $y_{i}$ more for larger $d_{i}$. The CHB estimates are sligtly larger than the empirical HB estimates. The risk estimates $R_{H B}$ and $R_{H B}^{*}$ are close each other, which may suggest that the parametric bootstrap estimates $R_{H B}^{*}$ are not bad. It is also revealed that the estimation errors of the empirical HB estimator for Chiba and Kanagawa are large, while those for Tokyo is small since $n_{i}$ is large and $d_{i}$ is small for Tokyo. The estimation errors $R_{C H B}^{*}$ of the empirical CHB estimator are slightly larger than those of the empirical HB estimator, and the estimator $R_{C H B}^{*}$ works well. 
Table 3: Values of HB and CHB with their risk estimates

\begin{tabular}{crr|rrr|rrr}
\hline Prefecture & $n_{i}$ & $d_{i}$ & $y_{i}$ & HB & CHB & $R_{H B}$ & $R_{H B}^{*}$ & $R_{C H B}^{*}$ \\
\hline Ibaraki & 95 & 9.56 & 8.10 & 8.88 & 9.66 & 19.5 & 19.1 & 22.4 \\
Tochigi & 95 & 26.96 & 10.03 & 9.47 & 10.30 & 23.6 & 23.4 & 27.3 \\
Gunma & 94 & 8.41 & 5.21 & 7.70 & 8.38 & 19.7 & 19.4 & 22.3 \\
Saitama & 95 & 3.93 & 12.33 & 12.72 & 13.83 & 19.9 & 19.7 & 25.0 \\
Chiba & 94 & 37.83 & 30.71 & 13.05 & 14.20 & 32.1 & 31.5 & 37.0 \\
Tokyo & 386 & 2.30 & 15.45 & 14.44 & 15.70 & 12.4 & 12.7 & 18.2 \\
Kanagawa & 142 & 15.99 & 23.25 & 14.02 & 15.25 & 30.0 & 29.3 & 35.0 \\
\hline \hline
\end{tabular}

\section{Concluding Remarks}

In this paper, we have considered the benchmark issue in the log-transformed Fay-Herriot multiplicative model for analyzing positive data like income, revenue and others, and we have derived the constrained hierarchical empirical Bayes (CHBEB) estimator relative to the weighted Kullback-Leibler loss function. Although the constrained Bayes estimators under some typical loss functions have difficulties from an aspect of practical use, the empirical CHB estimator under the weighted Kullback-Leibler loss is expressed as a simple and natural ratio-type estimator. We have derived not only the second-order approximation of the risk of the empirical hierarchical Bayes (HB) estimator, but also the second-order unbiased estimator of the risk via two approaches, namely, the analytical method based on Taylor series expansion and the numerical approach based on the parametric bootstrap.

It is important to point out that the asymptotic risk difference between the empirical $\mathrm{HB}$ and $\mathrm{CHB}$ estimators in the multiplicative model appears in the first-order term, while the corresponding difference in the additive model appears in the second-order term as shown in Steorts and Ghosh (2013). This fact suggests that the difference between the empirical $\mathrm{HB}$ and $\mathrm{CHB}$ estimators is not negligible, and we need to evaluate how much the risk of the empirical CHB estimator is inflated. We have provided the second-order unbiased estimator of the risk of the empirical CHB estimator based on the parametric bootstrap. The performances of the proposed procedures have been investigated through the empirical study, and it has been confirmed that the second-order unbiased estimators of the risks of the empirical $\mathrm{HB}$ and $\mathrm{CHB}$ estimators work well.

It may be meaningful to note that the weighted Kullback-Leibler loss is close to the quadratic loss functions $L_{Q}$ and $L_{T Q}$ in the neighborhood of $\hat{\theta}_{i}-\theta_{i}=0$. In fact, the Taylor series expansion tells us that it can be approximated as

$$
L_{m K L}\left(\theta_{i}, \hat{\theta}_{i}\right)=\left(\theta_{i} \log \left(\theta_{i} / \hat{\theta}_{i}\right)+\hat{\theta}_{i}-\theta_{i}=\left\{\begin{array}{l}
\left(\hat{\theta}_{i}-\theta_{i}\right)^{2} /\left(2 \theta_{i}\right)+o_{p}\left(\left(\hat{\theta}_{i}-\theta_{i}\right)^{2}\right) \\
\theta_{i}\left(\log \hat{\theta}_{i}-\log \theta_{i}\right)^{2} / 2+o_{p}\left(\left\{\log \left(\hat{\theta}_{i} / \theta_{i}\right)\right\}^{2}\right) .
\end{array}\right.\right.
$$


However, the $L_{Q}$-loss is quite different from the $L_{m K L^{-}}$-loss and $L_{T Q}$-loss when $\hat{\theta}_{i}$ is close to 0 . That is, $\lim _{\hat{\theta}_{i} \rightarrow 0}\left(\hat{\theta}_{i}-\theta_{i}\right)^{2}=\theta_{i}^{2}$, while $\theta_{i} \log \left(\theta_{i} / \hat{\theta}_{i}\right)+\hat{\theta}_{i}-\theta_{i}$ and $\left(\log \hat{\theta}_{i}-\log \theta_{i}\right)^{2}$ go to infinity as $\hat{\theta}_{i} \rightarrow 0$.

This is the first article that considers the benchmark issue in the simple multiplicative model, namely the transformed Fay-Herriot model. As future projects, one can extend the results to the unit-level multiplicative models and to the case where the benchmark constraint is a geometric mean. It is also interesting to consider the problem of constructing a confidence interval of $\theta_{i}$ with second-order accuracy.

\section{A Appendix}

\section{A.1 Proof of Theorem 3.1}

We begin by providing the following lemmas which will be used for the proof.

Lemma A.1 $\boldsymbol{x}_{i}^{T} \widehat{\boldsymbol{\beta}}\left(\tau^{2}\right)$ and $z_{i}-\boldsymbol{x}_{i}^{T} \widehat{\boldsymbol{\beta}}\left(\tau^{2}\right)$ are independently distributed as $\boldsymbol{x}_{i}^{T} \widehat{\boldsymbol{\beta}}\left(\tau^{2}\right) \sim \mathcal{N}\left(\boldsymbol{x}_{i}^{T} \boldsymbol{\beta}, g_{i}\right)$ and $z_{i}-\boldsymbol{x}_{i}^{T} \widehat{\boldsymbol{\beta}}\left(\tau^{2}\right) \sim \mathcal{N}\left(0, d_{i}+\tau^{2}-g_{i}\right)$, where $g_{i}=\boldsymbol{x}_{i}^{t}\left(\boldsymbol{X}^{T} \boldsymbol{\Sigma} \boldsymbol{X}\right)^{1} \boldsymbol{x}-i, i=1, \ldots, m$.

Lemma A.2 If $Y \sim \mathcal{N}\left(\mu, \sigma^{2}\right)$, then $E[Y \exp \{a Y\}]=\left(\mu+a \sigma^{2}\right) \exp \left\{a \mu+a^{2} \sigma^{2} / 2\right\}$.

The risk function of $\hat{\theta}_{i}^{H B}\left(\hat{\tau}^{2}\right)$ is written as

$$
\begin{aligned}
R_{\omega}\left(\hat{\theta}_{i}^{H B}\left(\hat{\tau}^{2}\right)\right)= & E\left[\hat{\theta}_{i}^{H B}\left(\hat{\tau}^{2}\right)-\theta_{i}-\theta_{i} \log \left\{\hat{\theta}_{i}^{H B}\left(\hat{\tau}^{2}\right) / \theta_{i}\right\}\right] \\
= & E\left[\left\{\hat{\theta}_{i}^{H B}\left(\tau^{2}\right)-\theta_{i}\right\}+\left\{\theta_{i} \log \theta_{i}-\theta_{i} \log \hat{\theta}_{i}^{B}\right\}-\theta_{i} \log \left\{\hat{\theta}_{i}^{H B}\left(\tau^{2}\right) / \hat{\theta}_{i}^{B}\right\}\right] \\
& +E\left[\hat{\theta}_{i}^{H B}\left(\hat{\tau}^{2}\right)-\hat{\theta}_{i}^{H B}\left(\tau^{2}\right)-\theta_{i} \log \left\{\hat{\theta}_{i}^{H B}\left(\hat{\tau}^{2}\right) / \hat{\theta}_{i}^{H B}\left(\tau^{2}\right)\right\}\right] \\
= & I_{1}+I_{2} . \quad \text { (say) }
\end{aligned}
$$

First, we shall evaluate each term in $I_{1}$. Note that $E\left[\theta_{i}\right]=E\left[\exp \left\{\phi_{i}\right\}\right]=\exp \left\{\boldsymbol{x}_{i}^{T} \boldsymbol{\beta}+\tau^{2} / 2\right\}=$ $h_{i}(\omega)$. Let $g_{i}=g_{i}\left(\tau^{2}\right)=\boldsymbol{x}_{i}^{T}\left(\boldsymbol{X}^{T} \boldsymbol{\Sigma}^{-1} \boldsymbol{X}\right)^{-1} \boldsymbol{x}_{i}$ and write $\widehat{\boldsymbol{\beta}}=\widehat{\boldsymbol{\beta}}\left(\tau^{2}\right)$. By Lemma A.1,

$$
\begin{aligned}
E\left[\hat{\theta}_{i}^{H B}\left(\tau^{2}\right)\right] & =E\left[\exp \left\{\boldsymbol{x}_{i}^{T} \widehat{\boldsymbol{\beta}}+\left(1-\gamma_{i}\right)\left(z_{i}-\boldsymbol{x}_{i}^{T} \widehat{\boldsymbol{\beta}}\right)+\tau^{2} \gamma_{i} / 2+\gamma_{i}^{2} g_{i} / 2\right\}\right] \\
& =\exp \left\{\boldsymbol{x}_{i}^{T} \boldsymbol{\beta}+g_{i} / 2+\left(1-\gamma_{i}\right)^{2}\left(d_{i}+\tau^{2}-g_{i}\right) / 2+\tau^{2} \gamma_{i} / 2+\gamma_{i}^{2} g_{i} / 2\right\} \\
& =h_{i}(\omega) \exp \left\{\gamma_{i} g_{i}\right\}
\end{aligned}
$$

so that one gets

$$
E\left[\hat{\theta}_{i}^{H B}\left(\tau^{2}\right)-\theta_{i}\right]=h_{i}(\omega)\left[\exp \left\{\gamma_{i} g_{i}\right\}-1\right]=h_{i}(\omega)\left[\gamma_{i} g_{i}+O\left(m^{-2}\right)\right] .
$$

It is noted that the conditional distribution of $\phi_{i}$ given $\boldsymbol{z}$ for fixed $\boldsymbol{\beta}$ and $\tau^{2}$ is distributed as $\phi_{i} \mid \boldsymbol{z} \sim \mathcal{N}\left(\widehat{\phi}_{i}^{B}, \tau^{2} \gamma_{i}\left(\tau^{2}\right)\right)$ for $\widehat{\phi}_{i}^{B}=\widehat{\phi}_{i}^{B}(\omega)=E\left[\phi_{i} \mid \boldsymbol{z}\right]=\left(1-\gamma_{i}\right) z_{i}+\gamma_{i} \boldsymbol{x}_{i}^{T} \boldsymbol{\beta}$. By Lemma A.2, it is seen that

$$
\begin{aligned}
E\left[\theta_{i} \log \theta_{i}\right] & =E\left[\phi_{i} \exp \left(\phi_{i}\right)\right]=E\left[E\left[\phi_{i} \exp \left(\phi_{i}\right) \mid \boldsymbol{z}\right]\right] \\
& =E\left[\left\{\widehat{\phi}_{i}^{B}+\tau^{2} \gamma_{i}\right\} \exp \left\{\widehat{\phi}_{i}^{B}+\tau^{2} \gamma_{i} / 2\right\}\right] \\
& =E\left[\hat{\theta}_{i}^{B}\left\{\widehat{\phi}_{i}^{B}+\tau^{2} \gamma_{i}\right\}\right] .
\end{aligned}
$$


Again, by Lemma A.2, $E\left[\theta_{i} \log \hat{\theta}_{i}^{B}\right]=E\left[E\left[\theta_{i} \mid \boldsymbol{z}\right] \log \hat{\theta}_{i}^{B}\right]=E\left[\hat{\theta}_{i}^{B}\left\{\widehat{\phi}_{i}^{B}+\tau^{2} \gamma_{i} / 2\right\}\right]$. Hence,

$$
E\left[\theta_{i} \log \theta_{i}-\theta_{i} \log \hat{\theta}_{i}^{B}\right]=2^{-1} \tau^{2} \gamma_{i} E\left[\hat{\theta}_{i}^{B}\right]=\tau^{2} \gamma_{i} h_{i}(\omega) / 2 .
$$

For the last term in $I_{1}$, note that $\hat{\theta}_{i}^{H B}\left(\tau^{2}\right)=\exp \left\{\left(1-\gamma_{i}\right) z_{i}+\gamma_{i} \boldsymbol{x}_{i}^{T} \boldsymbol{\beta}+\tau^{2} \gamma_{i} / 2+\gamma_{i} \boldsymbol{x}_{i}^{T}\left(\widehat{\boldsymbol{\beta}}\left(\tau^{2}\right)-\right.\right.$ $\left.\boldsymbol{\beta})+\gamma_{i}^{2} g_{i} / 2\right\}=\hat{\theta}_{i}^{B} \exp \left\{\gamma_{i} \boldsymbol{x}_{i}^{T}\left(\widehat{\boldsymbol{\beta}}\left(\tau^{2}\right)-\boldsymbol{\beta}\right)+\gamma_{i}^{2} g_{i} / 2\right\}$. Then,

$$
\begin{aligned}
E[ & \left.\theta_{i} \log \left\{\hat{\theta}_{i}^{H B}\left(\tau^{2}\right) / \hat{\theta}_{i}^{B}\right\}\right]=E\left[\hat{\theta}_{i}^{B} \log \left\{\hat{\theta}_{i}^{H B}\left(\tau^{2}\right) / \hat{\theta}_{i}^{B}\right\}\right]=E\left[\hat{\theta}_{i}^{B}\left\{\gamma_{i} \boldsymbol{x}_{i}^{T}\left(\widehat{\boldsymbol{\beta}}\left(\tau^{2}\right)-\boldsymbol{\beta}\right)+\gamma_{i}^{2} g_{i} / 2\right\}\right] \\
= & E\left[\exp \left\{\left(1-\gamma_{i}\right) z_{i}+\gamma_{i} \boldsymbol{x}_{i}^{T} \boldsymbol{\beta}+\tau^{2} \gamma_{i} / 2\right\} \gamma_{i} \boldsymbol{x}_{i}^{T}(\widehat{\boldsymbol{\beta}}-\boldsymbol{\beta})\right]+\gamma_{i}^{2} g_{i} h_{i}(\omega) / 2 \\
= & E\left[\exp \left\{\left(1-\gamma_{i}\right)\left(z_{i}-\boldsymbol{x}_{i}^{T} \widehat{\boldsymbol{\beta}}\right)+\left(1-\gamma_{i}\right) \boldsymbol{x}_{i}^{T}(\widehat{\boldsymbol{\beta}}-\boldsymbol{\beta})+\boldsymbol{x}_{i}^{T} \boldsymbol{\beta}+\tau^{2} \gamma_{i} / 2\right\} \gamma_{i} \boldsymbol{x}_{i}^{T}(\widehat{\boldsymbol{\beta}}-\boldsymbol{\beta})\right] \\
& +\gamma_{i}^{2} g_{i} h_{i}(\omega) / 2 .
\end{aligned}
$$

By Lemmas A.1 and A.2, this can be evaluated as

$$
\begin{aligned}
& \gamma_{i} \exp \left\{\left(1-\gamma_{i}\right)^{2}\left(d_{i}+\tau^{2}-g_{i}\right) / 2+\tau^{2} \gamma_{i} / 2+\boldsymbol{x}_{i}^{T} \beta\right\} E\left[\exp \left\{\left(1-\gamma_{i}\right) \boldsymbol{x}_{i}^{T}(\widehat{\boldsymbol{\beta}}-\boldsymbol{\beta})\right\} \boldsymbol{x}_{i}^{T}(\widehat{\boldsymbol{\beta}}-\boldsymbol{\beta})\right] \\
& \quad+\gamma_{i}^{2} g_{i} h_{i}(\omega) / 2 \\
& =\gamma_{i} \exp \left\{\boldsymbol{x}_{i}^{T} \boldsymbol{\beta}+\tau^{2}\left(1-\gamma_{i}\right) / 2-g_{i}\left(1-\gamma_{i}\right)^{2} / 2+\tau^{2} \gamma_{i} / 2\right\}\left(1-\gamma_{i}\right) g_{i} \exp \left\{\left(1-\gamma_{i}\right)^{2} g_{i} / 2\right\} \\
& \quad+\gamma_{i}^{2} g_{i} h_{i}(\omega) / 2 \\
& =h_{i}(\omega) \gamma_{i}\left(1-\gamma_{i}\right) g_{i}+\gamma_{i}^{2} g_{i} h_{i}(\omega) / 2=\gamma_{i} g_{i}\left(2-\gamma_{i}\right) h_{i}(\omega) / 2
\end{aligned}
$$

Combining (A.2)-(A.4), one gets

$$
I_{1}=h_{i}(\omega) \gamma_{i}\left(\tau^{2}+\gamma_{i} g_{i}\right) / 2+O\left(m^{-2}\right) .
$$

We next evaluate $I_{2}$. Write $k_{i}=k_{i}\left(\tau^{2}\right)=\tau^{2} \gamma_{i}+\gamma_{i}^{2} g_{i}$. It is noted that

$$
\hat{\theta}_{i}^{H B}\left(\hat{\tau}^{2}\right)-\hat{\theta}_{i}^{H B}\left(\tau^{2}\right)=\left(\hat{\tau}^{2}-\tau^{2}\right)\left(\hat{\theta}_{i}^{H B}\right)^{\prime}\left(\tau^{2}\right)+2^{-1}\left(\hat{\tau}^{2}-\tau^{2}\right)^{2}\left(\hat{\theta}_{i}^{H B}\right)^{\prime \prime}\left(\tau^{2}\right)+O_{p}\left(m^{-3 / 2}\right) .
$$

Since $\hat{\theta}_{i}^{H B}\left(\tau^{2}\right)=\exp \left\{\widehat{\phi}_{i}^{H B}\left(\tau^{2}\right)+k_{i}\left(\tau^{2}\right) / 2\right\}$, it is observed that $\left(\hat{\theta}_{i}^{H B}\right)^{\prime}\left(\tau^{2}\right)=\hat{\theta}_{i}^{H B}\left(\tau^{2}\right)\left\{\left(\widehat{\phi}_{i}^{H B}\right)^{\prime}\left(\tau^{2}\right)+\right.$ $\left.2^{-1} k_{i}^{\prime}\left(\tau^{2}\right)\right\}$ and

$$
\left(\hat{\theta}_{i}^{H B}\right)^{\prime \prime}\left(\tau^{2}\right)=\hat{\theta}_{i}^{H B}\left(\tau^{2}\right)\left\{\left(\widehat{\phi}_{i}^{H B}\right)^{\prime}\left(\tau^{2}\right)+2^{-1} k_{i}^{\prime}\left(\tau^{2}\right)\right\}^{2}+\hat{\theta}_{i}^{H B}\left(\tau^{2}\right)\left\{\left(\widehat{\phi}_{i}^{H B}\right)^{\prime \prime}\left(\tau^{2}\right)+2^{-1} k_{i}^{\prime \prime}\left(\tau^{2}\right)\right\} .
$$

Hence,

$$
\begin{aligned}
\hat{\theta}_{i}^{H B}\left(\hat{\tau}^{2}\right)-\hat{\theta}_{i}^{H B}\left(\tau^{2}\right)= & \left(\hat{\tau}^{2}-\tau^{2}\right) \hat{\theta}_{i}^{H B}\left(\tau^{2}\right)\left\{\left(\widehat{\phi}_{i}^{H B}\right)^{\prime}\left(\tau^{2}\right)+2^{-1} k_{i}^{\prime}\left(\tau^{2}\right)\right\} \\
& +2^{-1}\left(\hat{\tau}^{2}-\tau^{2}\right)^{2} \hat{\theta}_{i}^{H B}\left(\tau^{2}\right)\left\{\left(\widehat{\phi}_{i}^{H B}\right)^{\prime}\left(\tau^{2}\right)+2^{-1} k_{i}^{\prime}\left(\tau^{2}\right)\right\}^{2} \\
& +2^{-1}\left(\hat{\tau}^{2}-\tau^{2}\right)^{2} \hat{\theta}_{i}^{H B}\left(\tau^{2}\right)\left\{\left(\widehat{\phi}_{i}^{H B}\right)^{\prime \prime}\left(\tau^{2}\right)+2^{-1} k_{i}^{\prime \prime}\left(\tau^{2}\right)\right\}+O_{p}\left(m^{-3 / 2}\right) .
\end{aligned}
$$

Also, it is seen that

$$
\begin{aligned}
& E\left[\theta_{i} \log \left\{\hat{\theta}_{i}^{H B}\left(\hat{\tau}^{2}\right) / \hat{\theta}_{i}^{H B}\left(\tau^{2}\right)\right\}\right]=E\left[\hat{\theta}_{i}^{B} \log \left\{\hat{\theta}_{i}^{H B}\left(\hat{\tau}^{2}\right) / \hat{\theta}_{i}^{H B}\left(\tau^{2}\right)\right\}\right] \\
& =E\left[\hat{\theta}_{i}^{H B}\left(\tau^{2}\right) \log \left\{\frac{\hat{\theta}_{i}^{H B}\left(\hat{\tau}^{2}\right)}{\hat{\theta}_{i}^{H B}\left(\tau^{2}\right)}\right\}\right]+E\left[\left\{\hat{\theta}_{i}^{B}-\hat{\theta}_{i}^{H B}\left(\tau^{2}\right)\right\} \log \left\{\frac{\hat{\theta}_{i}^{H B}\left(\hat{\tau}^{2}\right)}{\hat{\theta}_{i}^{H B}\left(\tau^{2}\right)}\right\}\right] .
\end{aligned}
$$


Note that

$$
\begin{aligned}
\widehat{\phi}_{i}^{H B}\left(\hat{\tau}^{2}\right) & -\widehat{\phi}_{i}^{H B}\left(\tau^{2}\right)+\frac{1}{2} k_{i}\left(\hat{\tau}^{2}\right)-\frac{1}{2} k_{i}\left(\tau^{2}\right) \\
= & \left(\hat{\tau}^{2}-\tau^{2}\right)\left\{\left(\widehat{\phi}_{i}^{H B}\right)^{\prime}\left(\tau^{2}\right)+2^{-1} k_{i}^{\prime}\left(\tau^{2}\right)\right\} \\
& +2^{-1}\left(\hat{\tau}^{2}-\tau^{2}\right)^{2}\left\{\left(\widehat{\phi}_{i}^{H B}\right)^{\prime \prime}\left(\tau^{2}\right)+2^{-1} k_{i}^{\prime \prime}\left(\tau^{2}\right)\right\}+O_{p}\left(m^{-3 / 2}\right) .
\end{aligned}
$$

Hence, from (A.6)-(A.8),

$$
\begin{aligned}
I_{1}= & \frac{1}{2} E\left[\left(\hat{\tau}^{2}-\tau^{2}\right)^{2} \hat{\theta}_{i}^{H B}\left(\tau^{2}\right)\left\{\left(\widehat{\phi}_{i}^{H B}\right)^{\prime}\left(\tau^{2}\right)+2^{-1} k_{i}^{\prime}\left(\tau^{2}\right)\right\}^{2}\right] \\
& +E\left[\left\{\hat{\theta}_{i}^{B}-\hat{\theta}_{i}^{H B}\left(\tau^{2}\right)\right\} \log \left\{\frac{\hat{\theta}_{i}^{H B}\left(\hat{\tau}^{2}\right)}{\hat{\theta}_{i}^{H B}\left(\tau^{2}\right)}\right\}\right]+O\left(m^{-3 / 2}\right) .
\end{aligned}
$$

We shall approximate each term in (A.9). It can be seen that

$$
\begin{aligned}
E & {\left[\left(\hat{\tau}^{2}-\tau^{2}\right)^{2} \hat{\theta}_{i}^{H B}\left(\tau^{2}\right)\left\{\left(\widehat{\phi}_{i}^{H B}\right)^{\prime}\left(\tau^{2}\right)+2^{-1} k_{i}^{\prime}\left(\tau^{2}\right)\right\}^{2}\right] } \\
= & E\left[\left(\hat{\tau}^{2}-\tau^{2}\right)^{2}\right] E\left[\hat{\theta}_{i}^{H B}\left(\tau^{2}\right)\left\{\left(\widehat{\phi}_{i}^{H B}\right)^{\prime}\left(\tau^{2}\right)+2^{-1} k_{i}^{\prime}\left(\tau^{2}\right)\right\}^{2}\right] \\
& +\operatorname{Cov}\left[\left(\hat{\tau}^{2}-\tau^{2}\right)^{2}, \hat{\theta}_{i}^{H B}\left(\tau^{2}\right)\left\{\left(\widehat{\phi}_{i}^{H B}\right)^{\prime}\left(\tau^{2}\right)+2^{-1} k_{i}^{\prime}\left(\tau^{2}\right)\right\}^{2}\right] \\
= & \left\{\operatorname{Var}\left(\hat{\tau}^{2}\right)+O\left(m^{-2}\right)\right\} E\left[\hat{\theta}_{i}^{H B}\left(\tau^{2}\right)\left\{\left(\widehat{\phi}_{i}^{H B}\right)^{\prime}\left(\tau^{2}\right)+2^{-1} k_{i}^{\prime}\left(\tau^{2}\right)\right\}^{2}\right]+O\left(m^{-3 / 2}\right) .
\end{aligned}
$$

Note that $\operatorname{Var}\left(\hat{\tau}^{2}\right)=O\left(m^{-1}\right)$. Next observe that

$$
\begin{aligned}
\left(\widehat{\phi}_{i}^{H B}\right)^{\prime}\left(\tau^{2}\right) & =\left(\gamma_{i}^{2} / d_{i}\right)\left(z_{i}-\boldsymbol{x}_{i}^{T} \widehat{\boldsymbol{\beta}}\left(\tau^{2}\right)\right)-\gamma_{i} \boldsymbol{x}_{i}^{T}\left(\boldsymbol{X}^{T} \boldsymbol{\Sigma}^{-1} \boldsymbol{X}\right)^{-1} \boldsymbol{X}^{T} \boldsymbol{\Sigma}^{-2}\left(\boldsymbol{z}-\boldsymbol{X} \widehat{\boldsymbol{\beta}}\left(\tau^{2}\right)\right) \\
& =\left(\gamma_{i}^{2} / d_{i}\right)\left(z_{i}-\boldsymbol{x}_{i}^{T} \widehat{\boldsymbol{\beta}}\left(\tau^{2}\right)\right)+O_{p}\left(m^{-1 / 2}\right), \\
k_{i}^{\prime}\left(\tau^{2}\right) & =\gamma_{i}^{2}+2 \gamma_{i} g_{i}+\gamma_{i}^{2} \boldsymbol{x}_{i}^{T}\left(\boldsymbol{X}^{T} \boldsymbol{\Sigma}^{-1} \boldsymbol{X}\right)^{-1} \boldsymbol{X}^{T} \boldsymbol{\Sigma}^{-2}\left(\boldsymbol{X}^{T} \boldsymbol{\Sigma}^{-1} \boldsymbol{X}\right)^{-1} \boldsymbol{x}_{i}=\gamma_{i}^{2}+O\left(m^{-1}\right) .
\end{aligned}
$$

This leads to $\left(\widehat{\phi}_{i}^{H B}\right)^{\prime}\left(\tau^{2}\right)+2^{-1} k_{i}^{\prime}\left(\tau^{2}\right)=\left(\gamma_{i}^{2} / d_{i}\right)\left(z_{i}-\boldsymbol{x}_{i}^{T} \widehat{\boldsymbol{\beta}}\left(\tau^{2}\right)\right)+2^{-1} \gamma_{i}^{2}+O_{p}\left(m^{-1 / 2}\right)$, so that

$$
\begin{aligned}
E & {\left[\hat{\theta}_{i}^{H B}\left(\tau^{2}\right)\left\{\left(\widehat{\phi}_{i}^{H B}\right)^{\prime}\left(\tau^{2}\right)+2^{-1} k_{i}^{\prime}\left(\tau^{2}\right)\right\}^{2}\right] } \\
= & \gamma_{i}^{4} E\left[\hat{\theta}_{i}^{H B}\left(\tau^{2}\right)\left\{\left(z_{i}-\boldsymbol{x}_{i}^{T} \widehat{\boldsymbol{\beta}}\left(\tau^{2}\right)\right) / d_{i}+2^{-1}\right\}^{2}\right]+O\left(m^{-3 / 2}\right) \\
= & \gamma_{i}^{4} E\left[\left\{\left(z_{i}-\boldsymbol{x}_{i}^{T} \widehat{\boldsymbol{\beta}}\left(\tau^{2}\right)\right) / d_{i}+2^{-1}\right\}^{2} \exp \left\{\boldsymbol{x}_{i}^{T} \widehat{\boldsymbol{\beta}}+\left(1-\gamma_{i}\right)\left(z_{i}-\boldsymbol{x}_{i}^{T} \widehat{\boldsymbol{\beta}}\right)\right\}\right]+O\left(m^{-3 / 2}\right) \\
= & \gamma_{i}^{4} E\left[\exp \left\{\boldsymbol{x}_{i}^{T} \widehat{\boldsymbol{\beta}}\right\}\right] E\left[\left\{\left(z_{i}-\boldsymbol{x}_{i}^{T} \widehat{\boldsymbol{\beta}}\left(\tau^{2}\right)\right) / d_{i}+2^{-1}\right\}^{2} \exp \left\{\left(1-\gamma_{i}\right)\left(z_{i}-\boldsymbol{x}_{i}^{T} \widehat{\boldsymbol{\beta}}\right)\right\}\right]+O\left(m^{-3 / 2}\right) \\
= & \gamma_{i}^{4} \exp \left\{\boldsymbol{x}_{i}^{T} \boldsymbol{\beta}+\tau^{2} \gamma_{i} / 2\right\} \\
& \times E\left[\left\{\left(z_{i}-\boldsymbol{x}_{i}^{T} \widehat{\boldsymbol{\beta}}\left(\tau^{2}\right)\right)^{2} / d_{i}^{2}+\left(z_{i}-\boldsymbol{x}_{i}^{T} \widehat{\boldsymbol{\beta}}\left(\tau^{2}\right)\right) / d_{i}+1 / 4\right\} \exp \left\{\left(1-\gamma_{i}\right)\left(z_{i}-\boldsymbol{x}_{i}^{T} \widehat{\boldsymbol{\beta}}\right)\right\}\right]+O\left(m^{-3 / 2}\right) .
\end{aligned}
$$


Now using the result that if $Y \sim \mathcal{N}\left(0, \sigma^{2}\right)$, for any constant $a, E\left[Y^{2} \exp \{a Y\}\right]=\left(a^{2} \sigma^{4}+\right.$ $\left.\sigma^{2}\right) \exp \left\{a^{2} \sigma^{2} / 2\right\}$ and Lemma A.2,

$$
\begin{aligned}
E & {\left[\exp \left\{\left(1-\gamma_{i}\right)\left(z_{i}-\boldsymbol{x}_{i}^{T} \widehat{\boldsymbol{\beta}}\left(\tau^{2}\right)\right)\right\}\left\{\left(z_{i}-\boldsymbol{x}_{i}^{T} \widehat{\boldsymbol{\beta}}\left(\tau^{2}\right)\right)^{2} / d_{i}^{2}+\left(z_{i}-\boldsymbol{x}_{i}^{T} \widehat{\boldsymbol{\beta}}\left(\tau^{2}\right)\right) / d_{i}+1 / 4\right\}\right] } \\
= & \exp \left\{2^{-1}\left(1-\gamma_{i}\right)^{2}\left(d_{i}+\tau^{2}-g_{i}\right)\right\} \\
& \times\left[\left(1-\gamma_{i}\right)^{2}\left(d_{i}+\tau^{2}-g_{i}\right)^{2} / d_{i}^{2}+\left(d_{i}+\tau^{2}-g_{i}\right) / d_{i}^{2}+\left(1-\gamma_{i}\right)\left(d_{i}+\tau^{2}-g_{i}\right) / d_{i}+1 / 4\right] \\
= & \exp \left\{\tau^{2}\left(1-\gamma_{i}\right) / 2\right\}\left[\tau^{4} / d_{i}^{2}+\left(\gamma_{i} d_{i}\right)^{-1}+\tau^{2} / d_{i}+1 / 4\right]+O\left(m^{-1 / 2}\right) \\
= & \exp \left\{\tau^{2}\left(1-\gamma_{i}\right) / 2\right\}\left(4 \gamma_{i}\right)^{-1}\left[\left(2-\gamma_{i}\right)^{2}+4 \gamma_{i} / d_{i}\right]+O\left(m^{-1 / 2}\right),
\end{aligned}
$$

since $\tau^{2} / d_{i}=\left(1-\gamma_{i}\right) / \gamma_{i}$. Then, one gets

$$
\begin{aligned}
& \frac{1}{2} E\left[\left(\hat{\tau}^{2}-\tau^{2}\right)^{2} \hat{\theta}_{i}^{H B}\left(\tau^{2}\right)\left\{\left(\widehat{\phi}_{i}^{H B}\right)^{\prime}\left(\tau^{2}\right)+2^{-1} k_{i}^{\prime}\left(\tau^{2}\right)\right\}^{2}\right] \\
& =\frac{\gamma_{i}^{2}}{8} \operatorname{Var}\left(\hat{\tau}^{2}\right) h_{i}(\omega)\left[\left(2-\gamma_{i}\right)^{2}+4 \gamma_{i} / d_{i}\right]+O\left(m^{-3 / 2}\right) .
\end{aligned}
$$

Finally, consider $\left\{\hat{\theta}_{i}^{B}-\hat{\theta}_{i}^{H B}\left(\tau^{2}\right)\right\} \log \left\{\hat{\theta}_{i}^{H B}\left(\hat{\tau}^{2}\right) / \hat{\theta}_{i}^{H B}\left(\tau^{2}\right)\right\}$, which is rewritten as

$$
\hat{\theta}_{i}^{B}\left[\exp \left\{\gamma_{i} \boldsymbol{x}_{i}^{T}(\widehat{\boldsymbol{\beta}}-\boldsymbol{\beta})\right\}-1\right]\left[\widehat{\phi}_{i}^{H B}\left(\hat{\tau}^{2}\right)-\widehat{\phi}_{i}^{H B}\left(\tau^{2}\right)+\left\{k_{i}\left(\hat{\tau}^{2}\right)-k_{i}\left(\tau^{2}\right)\right\} / 2\right] .
$$

It is noted that $\exp \left\{\gamma_{i} \boldsymbol{x}_{i}^{T}(\widehat{\boldsymbol{\beta}}-\boldsymbol{\beta})\right\}-1=\gamma_{i} \boldsymbol{x}_{i}^{T}(\widehat{\boldsymbol{\beta}}-\boldsymbol{\beta})+2^{-1} \gamma_{i}^{2} \boldsymbol{x}_{i}^{T}(\widehat{\boldsymbol{\beta}}-\boldsymbol{\beta})(\widehat{\boldsymbol{\beta}}-\boldsymbol{\beta})^{T} \boldsymbol{x}_{i}+O_{p}\left(m^{-3 / 2}\right)$ and that $\left(\widehat{\phi}_{i}^{H B}\right)^{\prime}\left(\hat{\tau}^{2}\right)=\gamma_{i}^{2}\left[\left(z_{i}-\boldsymbol{x}_{i}^{T} \widehat{\boldsymbol{\beta}}\right) / d_{i}+1 / 2\right\}+O_{p}\left(m^{-1}\right)$. Then from (A.8) and the independence of $\widehat{\boldsymbol{\beta}}$ with $\left(\hat{\tau}^{2}, z_{i}-\boldsymbol{x}_{i}^{T} \boldsymbol{\beta}\right)$, it follows that

$$
\begin{aligned}
& E\left[\left\{\hat{\theta}_{i}^{B}-\hat{\theta}_{i}^{H B}\left(\tau^{2}\right)\right\} \log \left\{\hat{\theta}_{i}^{H B}\left(\hat{\tau}^{2}\right) / \hat{\theta}_{i}^{H B}\left(\tau^{2}\right)\right\}\right] \\
& \quad=E\left[\exp \left\{\left(1-\gamma_{i}\right) \boldsymbol{x}_{i}^{T}(\widehat{\boldsymbol{\beta}}-\boldsymbol{\beta})\right\} \gamma_{i} \boldsymbol{x}_{i}^{T}(\widehat{\boldsymbol{\beta}}-\boldsymbol{\beta})\right] E\left[\left(\hat{\tau}^{2}-\tau^{2}\right)\left\{\left(z_{i}-\boldsymbol{x}_{i}^{T} \widehat{\boldsymbol{\beta}}\right) / d_{i}+1 / 2\right\}\right]+O\left(m^{-3 / 2}\right) \\
& \quad=\gamma_{i}\left(1-\gamma_{i}\right) g_{i} \exp \left\{\left(1-\gamma_{i}\right)^{2} g_{i} / 2\right\} E\left[\left(\hat{\tau}^{2}-\tau^{2}\right)\left\{\left(z_{i}-\boldsymbol{x}_{i}^{T} \widehat{\boldsymbol{\beta}}\right) / d_{i}+1 / 2\right\}\right]+O\left(m^{-3 / 2}\right), \quad \text { (A.14) }
\end{aligned}
$$

which is of order $O\left(m^{-3 / 2}\right)$. Combining (A.13) and (A.14) yields that

$$
I_{2}=\frac{\gamma_{i}^{2}}{8} \operatorname{Var}\left(\hat{\tau}^{2}\right) h_{i}(\omega)\left[\left(2-\gamma_{i}\right)^{2}+4 \gamma_{i} / d_{i}\right]+O\left(m^{-3 / 2}\right) .
$$

Therefore, combining (A.5) and (A.15), one gets (3.1).

\section{A.2 Proof of Lemma 3.1}

Recall that $m_{i}=m_{i}(\omega)=d_{i}\left(1-\gamma_{i}\right) h_{i}=\tau^{2} \gamma_{i} h_{i}$ for $h_{i}=h_{i}(\omega)=\exp \left\{\boldsymbol{x}_{i}^{T} \boldsymbol{\beta}+\tau^{2} / 2\right\}$. Then, $\partial m_{i} / \partial \boldsymbol{\beta}=m_{i} \boldsymbol{x}_{i}, \partial^{2} m_{i} / \partial \boldsymbol{\beta} \partial \boldsymbol{\beta}^{T}=m_{i} \boldsymbol{x}_{i} \boldsymbol{x}_{i}^{T}, \partial^{2} m_{i} / \partial \boldsymbol{\beta} \partial \tau^{2}=\left(\partial m_{i} / \partial \tau^{2}\right) \boldsymbol{x}_{i}, \partial m_{i} / \partial \tau^{2}=\gamma_{i}^{2} h_{i}+$ $\tau^{2} \gamma_{i} h_{i} / 2$ and

$$
\begin{aligned}
\partial^{2} m_{i} / \partial\left(\tau^{2}\right)^{2}= & -2\left(\gamma_{i}^{3} / d_{i}\right) h_{i}+\gamma_{i}^{2} h_{i} / 2+\gamma_{i} h_{i} / 2-\tau^{2}\left(\gamma_{i}^{2} / d_{i}\right) h_{i} / 2+\tau^{2} \gamma_{i} h_{i} / 4 \\
& =-2\left(\gamma_{i}^{3} / d_{i}\right) h_{i}+\gamma_{i}^{2} h_{i}+\tau^{2} \gamma_{i} h_{i} / 4
\end{aligned}
$$


Thus, it is observed that

$$
\begin{aligned}
m_{i}(\hat{\omega})= & m_{i}(\omega)+\boldsymbol{x}_{i}^{T}\left(\widehat{\boldsymbol{\beta}}\left(\hat{\tau}^{2}\right)-\boldsymbol{\beta}\right) m_{i}(\omega)+\left(\hat{\tau}^{2}-\tau^{2}\right) \frac{\partial m_{i}(\omega)}{\partial \tau^{2}} \\
& +\frac{1}{2}\left\{\boldsymbol{x}_{i}^{T}\left(\widehat{\boldsymbol{\beta}}\left(\hat{\tau}^{2}\right)-\boldsymbol{\beta}\right)\right\}^{2} m_{i}(\omega)+\frac{1}{2}\left(\hat{\tau}^{2}-\tau^{2}\right)^{2} \frac{\partial^{2} m_{i}(\omega)}{\partial\left(\tau^{2}\right)^{2}} \\
& +\frac{1}{2}\left(\widehat{\boldsymbol{\beta}}\left(\hat{\tau}^{2}\right)-\boldsymbol{\beta}\right)^{T}\left(\hat{\tau}^{2}-\tau^{2}\right) \frac{\partial m_{i}(\omega)}{\partial \boldsymbol{\beta} \partial \tau^{2}}+O_{p}\left(m^{-3 / 2}\right) \\
= & m_{i}(\omega)+\left\{\widehat{\boldsymbol{\beta}}\left(\hat{\tau}^{2}\right)-\widehat{\boldsymbol{\beta}}\left(\tau^{2}\right)+\widehat{\boldsymbol{\beta}}\left(\tau^{2}\right)-\boldsymbol{\beta}\right\} m_{i}(\omega) \\
& +\left(\hat{\tau}^{2}-\tau^{2}\right)\left(\gamma_{i}^{2} h_{i}+\frac{1}{2} \tau^{2} \gamma_{i} h_{i}\right)+\frac{1}{2}\left[\boldsymbol{x}_{i}^{T}\left\{\widehat{\boldsymbol{\beta}}\left(\hat{\tau}^{2}\right)-\widehat{\boldsymbol{\beta}}\left(\tau^{2}\right)+\widehat{\boldsymbol{\beta}}\left(\tau^{2}\right)-\boldsymbol{\beta}\right\}\right]^{2} m_{i}(\omega) \\
& +\frac{1}{2} \boldsymbol{x}_{i}^{T}\left\{\widehat{\boldsymbol{\beta}}\left(\hat{\tau}^{2}\right)-\widehat{\boldsymbol{\beta}}\left(\tau^{2}\right)+\widehat{\boldsymbol{\beta}}\left(\tau^{2}\right)-\boldsymbol{\beta}\right\}\left(\hat{\tau}^{2}-\tau^{2}\right) \frac{\partial m_{i}(\omega)}{\partial \tau^{2}}+O_{p}\left(m^{-3 / 2}\right) .
\end{aligned}
$$

Using the independence of $\hat{\tau}^{2}$ and $\widehat{\boldsymbol{\beta}}\left(\tau^{2}\right)$ and the fact that $E\left[\widehat{\boldsymbol{\beta}}\left(\hat{\tau}^{2}\right)-\boldsymbol{\beta}\right]=\mathbf{0}$, one gets

$$
\begin{aligned}
E\left[m_{i}(\hat{\omega})\right]= & m_{i}(\omega)+\frac{1}{2} \gamma_{i} h_{i}\left(\tau^{2}+2 \gamma_{i}\right) E\left[\hat{\tau}^{2}-\tau^{2}\right]+\frac{1}{2} E\left[\left[\boldsymbol{x}_{i}^{T}\left\{\widehat{\boldsymbol{\beta}}\left(\tau^{2}\right)-\boldsymbol{\beta}\right\}\right]^{2}\right]\left\{1+O\left(m^{-1 / 2}\right)\right\} m_{i}(\omega) \\
& +\frac{1}{2} \gamma_{i} h_{i}\left(-2 \gamma_{i}^{2} / d_{i}+\gamma_{i}+\tau^{2} / 4\right) E\left[\left(\hat{\tau}^{2}-\tau^{2}\right)^{2}\right]+O\left(m^{-3 / 2}\right),
\end{aligned}
$$

which leads to the second-order approximation of $E\left[m_{i}(\hat{\omega})\right]$ is given in Lemma 3.1.

\section{A.3 Proof of Lemma 5.1}

Let $s_{i}=\left(z_{i}-\boldsymbol{x}_{i}^{T} \boldsymbol{\beta}\right) / \sqrt{\tau^{2}+d_{i}}$ for $i=1, \ldots, m$, and let $\boldsymbol{s}=\left(s_{1}, \ldots, s_{m}\right)^{T}=\boldsymbol{\Sigma}^{-1 / 2}(\boldsymbol{z}-\boldsymbol{X} \boldsymbol{\beta})$ for $\boldsymbol{\Sigma}=\operatorname{diag}\left(d_{1}+\tau^{2}, \ldots, d_{m}+\tau^{2}\right)$. Since $\boldsymbol{s} \sim \mathcal{N}(\mathbf{0}, \boldsymbol{I})$, it is seen that $E\left[y_{i}\right]=E\left[e^{z_{i}}\right]=$ $e^{\boldsymbol{x}_{i}^{T} \boldsymbol{\beta}} E\left[e^{\sqrt{\tau^{2}+d_{i}} s_{i}}\right]=\exp \left\{\boldsymbol{x}_{i}^{T} \boldsymbol{\beta}+\left(\tau^{2}+d_{i}\right) / 2\right\}$. Since $z_{i}$ and $z_{j}$ are mutually independent for $i \neq j$, it is observed that

$$
\begin{aligned}
\operatorname{Var}\left(\sum_{i=1}^{m} w_{i} y_{i}\right) & =\sum_{i=1}^{m} w_{i}^{2} E\left[\left\{y_{i}-E\left[y_{i}\right]\right\}^{2}\right] \\
& \left.=\sum_{i=1}^{m} w_{i}^{2} e^{2 \boldsymbol{x}_{i}^{T} \boldsymbol{\beta}} E\left[\left(e^{\sqrt{\tau^{2}+d_{i}} s_{i}}-e^{\left(\tau^{2}+d_{i}\right) / 2}\right)^{2}\right]\right) \\
& =\sum_{i=1}^{m} w_{i}^{2} e^{2 \boldsymbol{x}_{i}^{T} \boldsymbol{\beta}+\tau^{2}+d_{i}}\left(e^{\tau^{2}+d_{i}}-1\right),
\end{aligned}
$$

which is of order $O\left(\mathrm{~m}^{-1}\right)$ from conditions (C3) and (C6). This implies the first approximation in Lemma 5.1.

For the second approximation in Lemma 5.1, note that $\hat{\tau}^{2}-\tau^{2}=O_{p}\left(m^{-1 / 2}\right)$ and $\hat{\gamma}_{i}-\gamma_{i}=$ $O_{p}\left(m^{-1 / 2}\right)$ from condition (C5) for $\hat{\gamma}_{i}=\gamma_{i}\left(\hat{\tau}^{2}\right)=d_{i} /\left(d_{i}+\hat{\tau}^{2}\right)$. Then, it can be seen that

$$
\begin{aligned}
\log \hat{\theta}_{i}^{H B}\left(\hat{\tau}^{2}\right)= & z_{i}-\hat{\gamma}_{i}\left(z_{i}-\boldsymbol{x}_{i}^{T} \widehat{\boldsymbol{\beta}}\left(\hat{\tau}^{2}\right)\right)+\hat{\tau}^{2} \hat{\gamma}_{i} / 2 \\
= & \left\{z_{i}-\gamma_{i}\left(z_{i}-\boldsymbol{x}_{i}^{T} \boldsymbol{\beta}\right)+\tau^{2} \gamma_{i} / 2\right\}+\left(\gamma_{i}-\hat{\gamma}_{i}\right)\left(z_{i}-\boldsymbol{x}_{i}^{T} \boldsymbol{\beta}-\tau^{2} / 2\right) \\
& +\left(\hat{\tau}^{2}-\tau^{2}\right) \gamma_{i} / 2+O_{p}\left(m^{-1}\right),
\end{aligned}
$$


so that we get the approximation as

$$
\begin{aligned}
\hat{\theta}_{i}^{H B}\left(\hat{\tau}^{2}\right) & =\hat{\theta}_{i}^{B}(\omega) \exp \left\{\left(\gamma_{i}-\hat{\gamma}_{i}\right)\left(z_{i}-\boldsymbol{x}_{i}^{T} \boldsymbol{\beta}-\tau^{2} / 2\right)+\left(\hat{\tau}^{2}-\tau^{2}\right) \gamma_{i} / 2+O_{p}\left(m^{-1}\right)\right\} \\
& =\hat{\theta}_{i}^{B}(\omega)\left\{1+\left(\gamma_{i}-\hat{\gamma}_{i}\right)\left(z_{i}-\boldsymbol{x}_{i}^{T} \boldsymbol{\beta}-\tau^{2} / 2\right)+\left(\hat{\tau}^{2}-\tau^{2}\right) \gamma_{i} / 2+O_{p}\left(m^{-1}\right)\right\} .
\end{aligned}
$$

Thus, $\hat{\theta}_{i}^{H B}\left(\hat{\tau}^{2}\right)$ can be approximated as

$$
\begin{aligned}
\sum_{i=1}^{m} w_{i} \hat{\theta}_{i}^{H B}\left(\hat{\tau}^{2}\right)= & \sum_{i=1}^{m} w_{i} h_{i}(\omega)+\sum_{i=1}^{m} w_{i}\left\{\hat{\theta}_{i}^{B}(\omega)-h_{i}(\omega)\right\} \\
& +\sum_{i=1}^{m} w_{i} \hat{\theta}_{i}^{B}(\omega)\left\{\left(\gamma_{i}-\hat{\gamma}_{i}\right)\left(z_{i}-\boldsymbol{x}_{i}^{T} \boldsymbol{\beta}-\tau^{2} / 2\right)+\left(\hat{\tau}^{2}-\tau^{2}\right) \gamma_{i} / 2\right\}+O_{p}\left(m^{-1}\right) .
\end{aligned}
$$

Since the third term in the r.h.s. of (A.17) is of order $O_{p}\left(m^{-1 / 2}\right)$, it is sufficient to show that

$$
\sum_{i=1}^{m} w_{i}\left\{\hat{\theta}_{i}^{B}(\omega)-h_{i}(\omega)\right\}=O_{p}\left(m^{-1 / 2}\right) .
$$

It is noted that $\log \hat{\theta}_{i}^{B}(\omega)$ is expressed as $\hat{\theta}_{i}^{B}(\omega)=\exp \left\{a_{i} s_{i}+\boldsymbol{x}_{i}^{T} \boldsymbol{\beta}+\tau^{2} \gamma_{i} / 2\right\}$ for $a_{i}=(1-$ $\left.\gamma_{i}\right) \sqrt{d_{i}+\tau^{2}}$, so that $E\left[\hat{\theta}_{i}^{B}(\omega)\right]=\exp \left\{a_{i}^{2} / 2+\boldsymbol{x}_{i}^{T} \boldsymbol{\beta}+\tau^{2} \gamma_{i} / 2\right\}=h_{i}(\omega)$. Thus, $\sum_{i=1}^{m} w_{i} E\left[\hat{\theta}_{i}^{B}(\omega)-\right.$ $\left.h_{i}(\omega)\right]=0$. Then the variance of $\sum_{i=1}^{m} w_{i} \hat{\theta}_{i}^{B}(\omega)$ is approximated as

$$
\begin{aligned}
\operatorname{Var}\left(\sum_{i=1}^{m} w_{i} \hat{\theta}_{i}^{B}(\omega)\right) & =\sum_{i=1}^{m} w_{i}^{2} \alpha_{i}^{2} E\left[\left\{e^{a_{i} s_{i}}-E\left[e^{a_{i} s_{i}}\right]\right\}^{2}\right] \\
& =\sum_{i=1}^{m} w_{i}^{2} \alpha_{i}^{2}\left\{E\left[e^{2 a_{i} s_{i}}\right]-\left(E\left[e^{a_{i} s_{i}}\right]\right)^{2}\right\},
\end{aligned}
$$

for $\alpha_{i}=\exp \left\{\boldsymbol{x}_{i}^{T} \boldsymbol{\beta}+\tau^{2} \gamma_{i} / 2\right\}$. It can be easily verified that

$$
\sum_{i=1}^{m} w_{i}^{2} \alpha_{i}^{2}\left\{E\left[e^{2 a_{i} s_{i}}\right]-\left(E\left[e^{a_{i} s_{i}}\right]\right)^{2}\right\}=O\left(m^{-1}\right),
$$

from condition (C6). Combining (A.17), (A.18) and (A.19) shows that $\sum_{i=1}^{m} w_{i} \hat{\theta}_{i}^{H B}\left(\tau^{2}\right)=$ $\sum_{j=1}^{m} w_{j} h_{i}(\omega)+O_{p}\left(m^{-1 / 2}\right)$.

\section{A.4 Proof of Theorem 5.1}

Let us consider $K_{3}=K_{3}(\omega)$ and $K_{3}^{*}$ given in (5.4) and (5.5). If $K_{3}(\omega)=O\left(\mathrm{~m}^{-1}\right)$, then $K_{3}(\hat{\omega})=$ $K_{3}(\omega)+O_{p}\left(m^{-3 / 2}\right)$. Thus, $K_{3}^{*}=K_{3}(\hat{\omega})+O_{p}\left(m^{-3 / 2}\right)$, so that $E\left[K_{3}^{*}\right]=E\left[K_{3}(\hat{\omega})\right]+O\left(m^{-3 / 2}\right)=$ $K_{3}(\omega)+O\left(m^{-3 / 2}\right)$. This implies that $K_{3}^{*}$ is an estimator of $K_{3}$ with $E\left[K_{3}^{*}\right]=K_{3}+O\left(m^{-3 / 2}\right)$. Hence, it is sufficient to show that $K_{3}(\omega)=O\left(m^{-1}\right)$.

We begin by expanding $\log \left(\sum_{i=1}^{m} w_{i} y_{i} / \sum_{j=1}^{m} w_{j} \hat{\theta}_{j}^{H B}\left(\hat{\tau}^{2}\right)\right)$ stochastically. The first approximation in Lemma 5.1 gives the expression as $\sum_{i=1}^{m} w_{i} y_{i}=B_{0}+B_{1}$, where $B_{0}=\sum_{i=1}^{m} w_{i} h_{i}(\omega) e^{d_{i} / 2}=$ $O(1)$ and

$$
B_{1}=\sum_{i=1}^{m} w_{i}\left\{y_{i}-h_{i}(\omega) e^{d_{i} / 2}\right\}=O_{p}\left(m^{-1 / 2}\right) .
$$


Also, from (A.17) and (A.19), it follows that $\sum_{i=1}^{m} w_{i} \hat{\theta}_{i}^{H B}\left(\hat{\tau}^{2}\right)=A_{0}+A_{1}+O_{p}\left(m^{-1}\right)$, where

$$
\begin{aligned}
& A_{0}=\sum_{i=1}^{m} w_{i} h_{i}(\omega)=O(1), \\
& A_{1}=\sum_{i=1}^{m} w_{i}\left\{\hat{\theta}_{i}^{B}(\omega)-h_{i}(\omega)\right\}+\sum_{i=1}^{m} w_{i} \hat{\theta}_{i}^{B}(\omega)\left\{\left(\gamma_{i}-\hat{\gamma}_{i}\right)\left(z_{i}-\boldsymbol{x}_{i}^{T} \boldsymbol{\beta}-\frac{\tau^{2}}{2}\right)+\left(\hat{\tau}^{2}-\tau^{2}\right) \frac{\gamma_{i}}{2}\right\},
\end{aligned}
$$

which is of order $O_{p}\left(m^{-1 / 2}\right)$. Using these expansions, we can get the approximations given by

$$
\begin{gathered}
\frac{\sum_{i=1}^{m} w_{i} y_{i}}{\sum_{i=1}^{m} w_{i} \hat{\theta}_{i}^{H B}\left(\hat{\tau}^{2}\right)}=\frac{B_{0}}{A_{0}}-\frac{B_{0}}{A_{0}^{2}} A_{1}+\frac{1}{A_{0}} B_{1}+O_{p}\left(m^{-1}\right), \\
\log \left(\frac{\sum_{i=1}^{m} w_{i} y_{i}}{\sum_{i=1}^{m} w_{i} \hat{\theta}_{i}^{H B}\left(\hat{\tau}^{2}\right)}\right)=\log \left(\frac{B_{0}}{A_{0}}\right)-\frac{1}{A_{0}} A_{1}+\frac{1}{B_{0}} B_{1}+O_{p}\left(m^{-1}\right) .
\end{gathered}
$$

For the term $\hat{\theta}_{i}^{B}(\omega)-\hat{\theta}_{i}^{H B}\left(\hat{\tau}^{2}\right)$, from (A.16), it follows that

$$
\hat{\theta}_{i}^{B}(\omega)-\hat{\theta}_{i}^{H B}\left(\hat{\tau}^{2}\right)=\hat{\theta}_{i}^{B}(\omega)\left\{-\left(\gamma_{i}-\hat{\gamma}_{i}\right)\left(z_{i}-\boldsymbol{x}_{i}^{T} \boldsymbol{\beta}-\tau^{2} / 2\right)-\left(\hat{\tau}^{2}-\tau^{2}\right) \gamma_{i} / 2\right\}+O_{p}\left(m^{-1}\right),
$$

which is of order $O_{p}\left(m^{-1 / 2}\right)$. Combining (A.20) and (A.21), we can approximate $K_{3}$ as

$$
\begin{aligned}
K_{3}= & -E\left[\hat{\theta}_{i}^{B}(\omega)\left\{\left(\gamma_{i}-\hat{\gamma}_{i}\right)\left(z_{i}-\boldsymbol{x}_{i}^{T} \boldsymbol{\beta}-\frac{\tau^{2}}{2}\right)+\left(\hat{\tau}^{2}-\tau^{2}\right) \frac{\gamma_{i}}{2}\right\}\left\{\log \left(\frac{B_{0}}{A_{0}}\right)-\frac{1}{A_{0}} A_{1}+\frac{1}{B_{0}} B_{1}\right\}\right] \\
& +O\left(m^{-3 / 2}\right) \\
= & -\log \left(\frac{B_{0}}{A_{0}}\right) E\left[\hat{\theta}_{i}^{B}(\omega)\left\{\left(\gamma_{i}-\hat{\gamma}_{i}\right)\left(z_{i}-\boldsymbol{x}_{i}^{T} \boldsymbol{\beta}-\frac{\tau^{2}}{2}\right)+\left(\hat{\tau}^{2}-\tau^{2}\right) \frac{\gamma_{i}}{2}\right\}\right]+O\left(m^{-1}\right) .
\end{aligned}
$$

It is noted that

$$
\gamma_{i}-\hat{\gamma}_{i}=\frac{d_{i}}{\left(d_{i}+\tau^{2}\right)^{2}}\left(\hat{\tau}^{2}-\tau^{2}\right)+O_{p}\left(m^{-1}\right) .
$$

Hence, for the proof of $K_{3}=O\left(m^{-1}\right)$, it suffices to show that

$$
\begin{aligned}
E\left[\hat{\theta}_{i}^{B}(\omega)\left(\hat{\tau}^{2}-\tau^{2}\right)\right] & =O\left(m^{-1}\right), \\
E\left[\hat{\theta}_{i}^{B}(\omega)\left(\hat{\tau}^{2}-\tau^{2}\right)\left(z_{i}-\boldsymbol{x}_{i}^{T} \boldsymbol{\beta}\right)\right] & =O\left(m^{-1}\right) .
\end{aligned}
$$

To show these properties, note that

$$
\hat{\theta}_{i}^{B} \exp \left\{-\frac{1}{2\left(d_{i}+\tau^{2}\right)}\left(z_{i}-\boldsymbol{x}_{i}^{T} \boldsymbol{\beta}\right)^{2}\right\}=h_{i}(\omega) \exp \left\{-\frac{1}{2\left(d_{i}+\tau^{2}\right)}\left(z_{i}-\boldsymbol{x}_{i}^{T} \boldsymbol{\beta}-\tau^{2}\right)^{2}\right\},
$$

where $h_{i}(\omega)=\exp \left\{\boldsymbol{x}_{i}^{T} \boldsymbol{\beta}+\tau^{2} / 2\right\}$. Thus, for a function $f(\boldsymbol{z})$ satisfying $E[|f(\boldsymbol{z})|]<\infty$,

$$
E\left[\hat{\theta}_{i}^{B} f(\boldsymbol{z})\right]=h_{i}(\omega) E\left[f\left(\boldsymbol{v}+\boldsymbol{X} \boldsymbol{\beta}+\tau^{2} \boldsymbol{e}_{i}\right)\right],
$$

where $\boldsymbol{v}$ is a random variable having $\mathcal{N}(\mathbf{0}, \boldsymbol{\Sigma})$, and $\boldsymbol{e}_{i}$ is an $m$-variate vector such that the $i$-th element is one and the others are zero. Then, we can rewrite the condition (A.22) as

$$
\begin{aligned}
E\left[\hat{\tau}^{2}\left(\boldsymbol{v}+\tau^{2} \boldsymbol{e}_{i}\right)-\tau^{2}\right] & =O\left(m^{-1}\right), \\
E\left[\left\{\hat{\tau}^{2}\left(\boldsymbol{v}+\tau^{2} \boldsymbol{e}_{i}\right)-\tau^{2}\right\}\left(v_{i}+\tau^{2}\right)\right] & =O\left(m^{-1}\right) .
\end{aligned}
$$


Here from the assumption of the theorem, it follows that $\hat{\tau}^{2}\left(\boldsymbol{z}+\tau^{2} \boldsymbol{e}_{i}\right)=\hat{\tau}^{2}(\boldsymbol{z})+O_{p}\left(m^{-1}\right)$ and $E\left[\hat{\tau}^{2}-\tau^{2}\right]=O\left(m^{-1}\right)$, which shows that $E\left[\hat{\tau}^{2}\left(\boldsymbol{v}+\tau^{2} \boldsymbol{e}_{i}\right)-\tau^{2}\right]=O\left(m^{-1}\right)$. Similarly, the second claim in (A.24) can be verified if $E\left[\left\{\hat{\tau}^{2}(\boldsymbol{v})-\tau^{2}\right\} v_{i}\right]=O\left(m^{-1}\right)$, or $E\left[\hat{\tau}^{2}(\boldsymbol{v}) v_{i}\right]=O\left(m^{-1}\right)$. It is verified that $E\left[\hat{\tau}^{2}(\boldsymbol{v}) v_{i}\right]=0$ from condition (C4). Hence, the sufficient conditions given in (A.24) are satisfied, and the proof of Theorem 5.1 is complete.

\section{Acknowledgments.}

Research of the first author was supported by NSF Grants SES-1026165 and SES1327359.Research of the second author was supported in part by Grant-in-Aid for Scientific Research (21540114 and 23243039) from Japan Society for the Promotion of Science.

\section{References}

[1] Bell, W.R., Datta, G.S. and Ghosh, M. (2013). Benchmarking small area estimators. Biometrika, 100, 189-202.

[2] Butar, F.B. and Lahiri, P. (2003). On measures of uncertainty of empirical Bayes small-area estimators. J. Statist. Plan. Inf., 112, 63-76.

[3] Datta, G.S., Ghosh, M., Steorts, R., and Maples, J. (2011). Bayesian benchmarking with applications to small area estimation. Test, 20, 574-588.

[4] Datta, G.S. and Lahiri, P. (2000). A unified measure of uncertainty of estimated best linear unbiased predictors in small area estimation problems. Statist. Sinica, 10, 613-627.

[5] Datta, G.S., Rao, J.N.K. and Smith, D.D. (2005). On measuring the variability of small area estimators under a basic area level model. Biometrika, 92, 183-196.

[6] Fay, R.E. and Herriot, R. (1979). Estimates of income for small places: An application of James-Stein procedures to census data. J. Amer. Statist. Assoc., 74, 269-277.

[7] Ghosh, M. (1992). Constrained Bayes estimation with applications. J. American Statist. Assoc., 87, 533-540.

[8] Ghosh, M. and Rao, J.N.K. (1994). Small area estimation: An appraisal. Statist. Science, 9, 55-93.

[9] Ghosh, M. and Steorts, R. (2013). Two-stage Bayesian benchmarking as applied to small area estimation. Test, 22, 670-687.

[10] Isaki, C.T., Tsay, J.H. and Fuller, W.A. (2000). Estimation of census adjustment factors. Survey Methodology, 31- 42. 
[11] James, W. and Stein, C. (1961). Estimation with quadratic loss. In Proc. Fourth Berkeley Symp. Math. Statist. Probab., 1, 361-379. University of California Press, Berkeley.

[12] Morris, C. N. (1983). Parametric empirical Bayes inference: theory and applications. J. Amer. Statist. Assoc., 78, 47-55.

[13] Prasad, N.G.N. and Rao, J.N.K. (1990). The estimation of the mean squared error of small-area estimators. J. Amer. Statist. Assoc., 85, 163-171.

[14] Pfeffermann, D. (2013). New important developments in small area estimation. Statist. Sci., 28, 1-134.

[15] Pfeffermann, D. and Barnard, C.H. (1991). Some new estimators for small area means with application to the assessment of farmland values. J. Business and Economic Statistics, 73-84.

[16] Rao, J.N.K. (2003). Small Area Estimation. Wiley.

[17] Steorts, R. and Ghosh, M. (2013). On estimation of mean squared errors of benchmarked empirical Bayes estimators. Statistica Sinica, 23, 749-767.

[18] Slud, E.V. and Maiti, T. (2006). Mean-squared error estimation in transformed Fay-Herriot models. J. Royal Statist. Soc., B 68, 239-257.

[19] Wang, J., Fuller, W.A. and Qu, Y. (2008). Small area estimation under a restriction. Survey Methodology, 29-36.

[20] You, Y. and Rao, J. N. K. (2002). A pseudo-empirical best linear unbiased prediction approach to small area estimation using survey weights. Canadian J. Statis., 30, 431-439.

[21] You, Y. and Rao, J.N.K. (2003). Pseudo hierarchical Bayes small area estimation combining unit level models and survey weights. J. Statist. Plann. Inf., 111, 197208. 